This item was submitted to Loughborough's Research Repository by the author.

Items in Figshare are protected by copyright, with all rights reserved, unless otherwise indicated.

\title{
Strong influence of decoherence corrections and momentum rescaling in surface hopping dynamics of transition metal complexes
}

\section{PLEASE CITE THE PUBLISHED VERSION}

\section{PUBLISHER}

American Chemical Society

\section{VERSION}

AM (Accepted Manuscript)

\section{PUBLISHER STATEMENT}

This document is the Accepted Manuscript version of a Published Work that appeared in final form in Journal of Chemical Theory and Computation, copyright @ American Chemical Society after peer review and technical editing by the publisher. To access the final edited and published work see https://pubs.acs.org/doi/10.1021/acs.jctc.9b00525.

\section{LICENCE}

CC BY-NC-ND 4.0

\section{REPOSITORY RECORD}

Plasser, Felix, Sebastian Mai, Maria Fumanal, Etienne Gindensperger, Chantal Daniel, and Leticia Gonzalez. 2019. "Strong Influence of Decoherence Corrections and Momentum Rescaling in Surface Hopping Dynamics of Transition Metal Complexes”. figshare. https://hdl.handle.net/2134/9205748.v1. 


\title{
Strong Influence of Decoherence Corrections and Momentum Rescaling in Surface Hopping Dynamics of Transition Metal Complexes
}

\author{
Felix Plasser, ${ }^{* \dagger \dagger}$, Sebastian Mai, ${ }^{\dagger}$ Maria Fumanal, ${ }^{\ddagger}$ Etienne Gindensperger, ${ }^{\ddagger}$ \\ Chantal Daniel, ${ }^{\ddagger}$ and Leticia González ${ }^{\dagger}$ \\ $\dagger$ Institute for Theoretical Chemistry, Faculty of Chemistry, University of Vienna, \\ Währingerstr. 17, 1090 Vienna, Austria \\ $\ddagger$ Laboratoire de Chimie Quantique, Institut de Chimie Strasbourg, UMR717n \\ CNRS/Université de Strasbourg \& Rue Blaise Pascal BP296/R8, F-67008 Strasbourg, \\ France \\ IDepartment of Chemistry, Loughborough University, Loughborough, LE11 3TU, U.K. \\ E-mail: f.plasser@lboro.ac.uk
}

\section{Abstract}

The reliability of different parameters in the surface hopping method is assessed for a vibronic coupling model of a challenging transition metal complex, where a large number of electronic states of different multiplicities are met within a small energy range. In particular, the effect of two decoherence correction schemes and of various strategies for momentum rescaling and treating frustrating hops during the dynamics is investigated and compared against an accurate quantum dynamics simulation. The results show that surface hopping is generally able to reproduce the reference but also that small differences in the protocol used can strongly affect the results. We find a clear preference for momentum rescaling along only one degree of freedom, using either the nonadiabatic coupling or the gradient difference vector, and trace this effect back to an enhanced number of frustrated hops. Furthermore, reflection of the momentum after frustrated hops is shown to work better than to ignore the process completely. The study also highlights the importance of the decoherence correction but neither of the two methods employed, en- ergy based decoherence or augmented fewest switches surface hopping, performs completely satisfactory and we trace this effect back to a lack of size-consistency. Finally, the effect of different methods for analysing the populations is highlighted. More generally, the study emphasises the importance of the often neglected parameters in surface hopping and shows that there is still need for simple, robust, and generally applicable correction schemes.

\section{Introduction}

Many important processes in photochemistry and electrochemistry are governed by nonadiabatic transitions between electronic states, ${ }^{1-8}$ at which the Born-Oppenheimer approximation breaks down, meaning that electronic and nuclear degrees of freedom can no longer be separated. The surface hopping dynamics method ${ }^{9}$ has become a popular approach to describe nonadiabatic processes due to its conceptual simplicity and the intuitive interpretation of the results in a quasi-classical picture. As a result, surface hopping is widely applied in many different application areas and and a large body of recent work exists. ${ }^{5,10-17}$ The simplicity of 
the classical picture is deceptive as it nevertheless needs to mimic non-trivial underlying quantum processes, such as (i) the branching of the wavepacket onto different electronic states, (ii) the loss of electronic coherence due to interactions with the nuclei or the environment, (iii) the exchange of energy and momentum between electronic and nuclear degrees of freedom, and (iv) classically forbidden transitions.

Point (i) is treated by the surface hopping algorithm itself meaning that rather than describing the whole wavepacket branching onto different potential energy surfaces (PES), one of the surfaces is selected by using a stochastic algorithm and only this branch is further propagated; an ensemble of trajectories following the different branches is then needed to resemble a bifurcating quantum wavepacket. This set-up naturally leads to some ambiguity with respect to the interpretation of the dynamics and different methods have been proposed. ${ }^{18}$ The fact that only one branch is propagated, automatically means that it is not possible to model the interactions between different branches and their eventual loss of coherence (ii), and this has led to the introduction of decoherence corrections on top of the surface hopping algorithm. ${ }^{19-23}$ An exchange of energy and momentum (iii) should occur during surface hops and different schemes of redistributing energy and momentum have been developed. Here, a new complication (iv) comes into play if the quantum and classical descriptions lead to incompatible results and the quantum propagation requires a classically forbidden hop, also called a "frustrated hop". ${ }^{21,24}$ In order to deal with the above-mentioned formal problems as well as additional numerical problems ${ }^{25}$ a number of different flavours of the surface hopping method have been developed, ${ }^{11,14,16,26}$ able to work under different circumstances.

While surface hopping simulations can depend strongly on the electronic structure method employed for the underlying on-thefly calculations, ${ }^{27,28}$ it is often forgotten that changes in the surface hopping algorithm can also have its consequences. The reliability of surface hopping algorithms has been tested particularly on idealised model systems, such as spin-boson models, ${ }^{29-31}$ a quantum oscillator, ${ }^{32}$ a two-level system in a classical bath, ${ }^{33}$ or on low-dimensional scattering problems ${ }^{34-36}$ and the one-dimensional $\mathrm{LiH}$ system. ${ }^{37}$ There also exist a few studies using realistic high-dimensional PES via on-the-fly dynamics, ${ }^{21,38-40}$ but in this case it is more challenging to find an accurate reference to compare with. Generally speaking, the validity of surface-hopping could be only assessed on simple systems where accurate reference values are available. In contrast, a reference for realistic large systems is much more difficult to obtain and it is of interest whether a lack of size-consistency in the protocol can have negative effects in larger systems that are not seen in smaller ones.

In this paper, we introduce a new and generally applicable strategy to assess the quality of surface hopping on complex large systems using high-dimensional, multi-state vibronic coupling models. Since their introduction in the $80 \mathrm{~s},{ }^{41}$ vibronic coupling models have been very successful $^{42-47}$ in reproducing experimental work, particularly in combination with the multiconfigurational time-dependent Hartree (MCTDH) method. ${ }^{48-50}$ Recently, we implemented an algorithm to perform surface hopping based on vibronic coupling models, ${ }^{51}$ and showed that it can be extremely cheap computationally while still capturing the main features of a variety of photophysical processes. Here, we shall use a linear vibronic coupling (LVC) model ${ }^{41,52}$ to compare the results of surface hopping against an accurate quantum dynamics reference for $\left[\operatorname{Re}(\mathrm{im})(\mathrm{CO})_{3}(\mathrm{phen})\right]^{+}(\mathrm{im}=$ imidazole, phen $=$ phenanthroline, see Fig. 1). ${ }^{53,54}$

The choice of a transition metal complex as a test bed is purposely, as such systems feature a high number of excited electronic states of different multiplicities in a limited domain of energy and the description of its dynamics represent a particularly challenging case for spin-vibronic models. ${ }^{55}$ Moreover, the dynamics of $\left[\operatorname{Re}(\mathrm{im})(\mathrm{CO})_{3}(\text { phen })\right]^{+}$is particularly rich, as due to the presence of an intermediate intra-ligand triplet state ${ }^{3} \mathrm{IL}\left(T_{3}\right.$ at the FranckCondon geometry) that couples strongly with the initially populated second singlet metal- 
to-ligand charge transfer ${ }^{1} \mathrm{MLCT}\left(S_{2}\right)$ state, spin-orbit coupling (SOC) effects are dominant at the early time of the dynamics $(<50 \mathrm{fs})$ while vibronic effects lead to populate the lowest ${ }^{3} \mathrm{MLCT}\left(T_{1}\right)$ state by exchange with the lowest ${ }^{3} \mathrm{IL}$ state. Depending on the character and relative positions of the low-lying states the early time spin-vibronic mechanism will be driven essentially either by vibronic effects ${ }^{46,56}$ or by SOC effects. ${ }^{53}$

Studies based on the LVC model performed on a series of rhenium (I) carbonyl $\alpha$-diimine complexes revealed the dominant normal modes and associated (spin) vibronic couplings that drive the ultra-fast decay $(<200 \mathrm{fs})$ within the low-lying singlet and triplet states. ${ }^{46,53,54,56,57}$ Previous simulations on $\left[\operatorname{Re}(\mathrm{im})(\mathrm{CO})_{3}(\text { phen })\right]^{+}$ showed that the key normal modes are the phen and carbonyl vibrations whereas the imidazole ligand is a spectator. ${ }^{53,58}$ Here we employ a model of 2 singlet and 4 triplet states, vibronically coupled via 15 normal modes, as well as three additional models with a reduced set of electronic states. In each case, 13 different surface hopping protocols are compared to the MCTDH reference. The 200 trajectories propagated over $500 \mathrm{fs}$ are equivalent to a total of more than ten million formal electronic structure computations. Doing this would be hardly feasible with on-the-fly dynamics but it requires negligible computational effort with our new implementation of $\mathrm{LVC}^{51}$ in the SHARC (surface hopping including arbitrary couplings) ${ }^{59,60}$ dynamics package. ${ }^{61}$

\section{Methods}

Here we review essential aspects of surface hopping, such as the representations for the electronic wavefunctions, the LVC approximation, and the methodological details of the surface hopping algorithm investigated in this work, i.e., decoherence corrections, momentum rescaling and frustrated hops. We proceed by discussing different ways to analyse and interpret the results and finish by listing the more technical computational details.

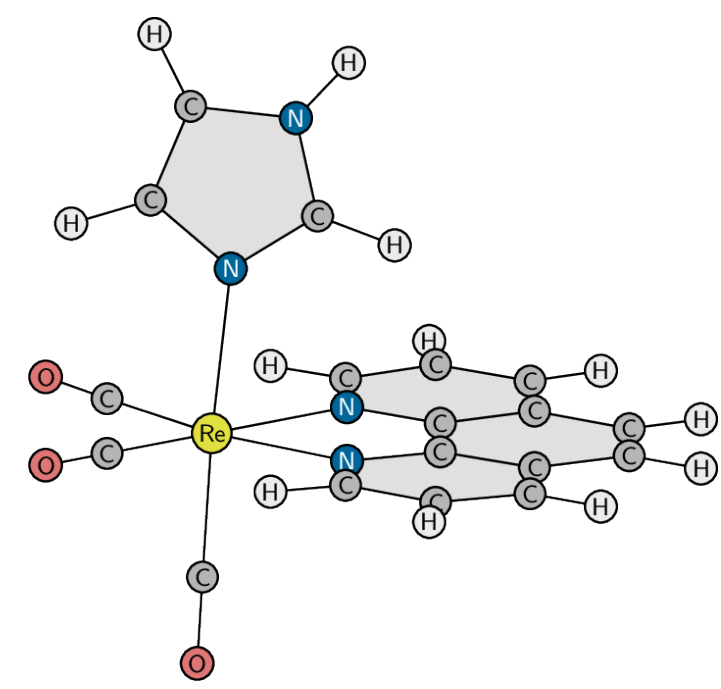

Figure 1: Chemical structure of the $\left[\operatorname{Re}(\mathrm{im})(\mathrm{CO})_{3}(\text { phen })\right]^{+}$complex studied within this work.

\subsection{Wavefunction representations}

An important ingredient of SHARC is the optimal use of the possible representations of the electronic wavefunctions. ${ }^{11}$ To ease the discussion, we establish here the name conventions employed. ${ }^{11,59,60}$ The three representations used are shown in Figure 2 (a-c), ordered such that the number of state crossings decreases from left to right. Most quantum chemistry codes work with an electronic Hamiltonian that includes molecular Coulomb interactions but neither external fields nor SOC. We label this operator the molecular Coulomb Hamiltonian $(\mathrm{MCH})$ and its eigenfunctions form the $\mathrm{MCH}$ basis (Figure 2 (b)). In this representation, states possessing distinct multiplicity are labelled as $S_{1}, S_{2}, \ldots, T_{1}, T_{2}, \ldots$. States of the same spin-multiplicity do not cross in a onedimensional picture whereas states of different multiplicities do. The MCH states can be transformed as to minimise kinetic couplings, leading to states of almost constant character, known as diabatic states ${ }^{41,52,62}$ (Figure $2(\mathrm{a})$ ) that are coupled by potential-like terms and can freely cross. These diabatic states are labelled according to their state character, e.g. $1^{1} \mathrm{MLCT}$ and $2^{1}$ MLCT. The Hamiltonian including SOC is termed the "total Hamiltonian" and its eigenfunctions, generally possessing mixed spin, are the basis of what we call the "diagonal" rep- 
resentation $^{59}$ (Figure 2 (c)). These states do not cross in a one-dimensional picture. It is important to realise that while the diabatic and $\mathrm{MCH}$ pictures feature a single (triply degenerate) PES for every triplet state, the diagonal representation considers explicitly the three individual surfaces arising from the triplet state.

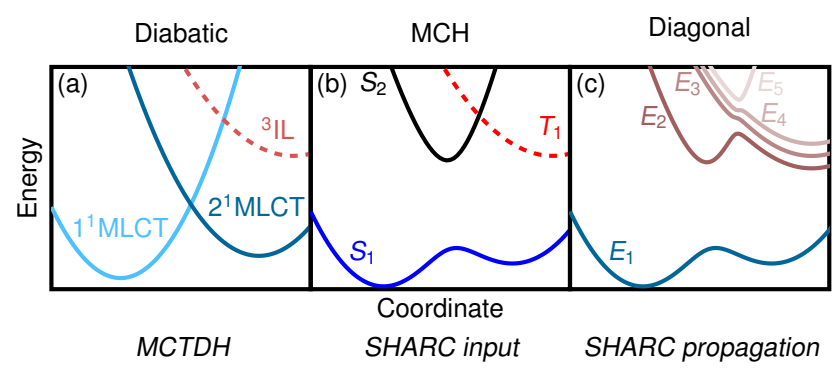

Figure 2: Wavefunction representations used in this work: (a) the diabatic representation, which is the basis for the LVC model and used for MCTDH dynamics, (b) the MCH representation, which is used by typical quantum chemistry codes and is the input for SHARC, and (c) the diagonal representation, which is used for SHARC propagations.

The LVC model (see Section 2.2) is constructed in a diabatic basis so that it can be directly used in MCTDH. ${ }^{50}$ In contrast, SHARC expects input in the $\mathrm{MCH}$ representation and propagates the wavefunction in the diagonal picture. It is, thus, necessary to transform the LVC states into the MCH representation before feeding this data into SHARC, as described in Ref. 51. The output from SHARC can be transformed back into any of the three pictures. In this way, it is possible to perform a one-to-one comparison between SHARC and MCTDH despite the fact that different representations are used for the wavefunction propagation.

\subsection{The linear vibronic coupling model}

Within a vibronic coupling model, ${ }^{41,52}$ the PES are constructed in the diabatic representation, cf. Fig. 2 (a), as

$$
\mathbf{V}=V_{0} \mathbf{1}+\mathbf{W}
$$

where $V_{0}$ is the ground state potential and the W matrix collects the state-specific vibronic coupling terms. The ground state potential is harmonic and given as

$$
V_{0}=\sum_{i} \frac{\hbar \omega_{i}}{2} Q_{i}^{2} .
$$

Here, $Q_{i}$ is a dimensionless mass-frequency scaled normal coordinate (cf. Ref. 52) defined as

$$
Q_{i}=\sqrt{\frac{\omega_{i}}{\hbar}} \sum_{\alpha} K_{\alpha i} \sqrt{M_{\alpha}} r_{\alpha},
$$

where $\omega_{i}$ is the frequency of normal mode $i, M_{\alpha}$ is an atomic mass, and $K_{\alpha i}$ denotes the orthogonal conversion matrix between mass-weighted Cartesian and normal coordinates.

Within the current work, a linear vibronic coupling model (LVC) is considered, which contains the following state-specific terms in the $\mathbf{W}$ matrix.

$$
\begin{aligned}
& W_{n n}=\epsilon_{n}+\sum_{i} \kappa_{i}^{(n)} Q_{i} \\
& W_{m n}=\sum_{i} \lambda_{i}^{(m, n)} Q_{i} .
\end{aligned}
$$

The $\epsilon_{n}$ are the vertical excitation energies. The $\kappa_{i}^{(n)}$ and $\lambda_{i}^{(m, n)}$ are termed intrastate and interstate vibronic coupling constants. ${ }^{41}$ Here these parameters were constructed from gradients and Hessian matrices, as described elsewhere, ${ }^{53,54}$ while we have also shown that wavefunction overlaps can be used effectively for this purpose. ${ }^{57}$ In addition, diabatic SOC constants were included as off-diagonal coupling terms, as outlined in Ref. 53. All quantities required by the SHARC dynamics program can be constructed on-the-fly by means of straightforward matrix operations, as detailed in Ref. 51.

\subsection{Decoherence corrections}

Decoherence is a fundamental concept in our understanding of how a system governed by the laws of quantum mechanics can effectively behave classically. ${ }^{63,64}$ In the context of surface hopping dynamics decoherence comes into play whenever the electronic wavepacket splits into 
two different PES. For illustration, let us consider an electronic wavepacket propagating on two coupled PES with different nuclear gradients, see Fig. 3. Initially, the components on the upper and lower surfaces start in the same region in space. However, while the component of the wavepacket on the upper surface moves at constant speed, the part on the lower surface accelerates. As a consequence, the two parts of the wavepacket no longer occupy the same region of space, leading to loss of coherence. If the system is simulated through surface hopping dynamics, only one branch of the wavepacket, for example the one on the upper surface, is explicitly propagated for each individual trajectory. The nuclear coordinates on the second surface, indicated by empty squares in Fig. 3, are artificially fixed to match those of the first branch. As a consequence, in standard surface hopping decoherence is not treated correctly and a decoherence correction is usually included. In this work, we examine the effect of two types of decoherence corrections. One is the energy based decoherence (EDC) scheme of Grannucci et al. ${ }^{21}$ - based on earlier work from Truhlar and co-workers ${ }^{19,20}$ - which only requires information about energies at the current time step. The other is a somewhat more involved formalism, denoted augmented fewest switches surface hopping (AFSSH) as introduced by Subotnik and co-workers. ${ }^{23,65}$ The essence of the AFSSH method is that it explicitly propagates auxiliary trajectories on the potential surfaces that are not active in the dynamics.

The EDC method ${ }^{21}$ proceeds by defining a decoherence time

$$
\tau_{\alpha \lambda}=\frac{\hbar}{\left|E_{\alpha}-E_{\lambda}\right|}\left(1+\frac{C}{E_{k i n}}\right)
$$

where $E_{\lambda}$ and $E_{\alpha}$ are the potential energies of the active surface $\lambda$ and any other state $\alpha, E_{k i n}$ is the kinetic energy, and $\mathrm{C}$ is an adjustable parameter usually set as $C=0.1 \mathrm{H}^{19}$ The decoherence time $\tau_{\alpha \lambda}$ is used to continuously damp the coefficients $c_{\alpha}$ of all non-active states in the diagonal representation (cf. Fig. 2 (c)) at each time step. To this aim, $c_{\alpha}$ is replaced

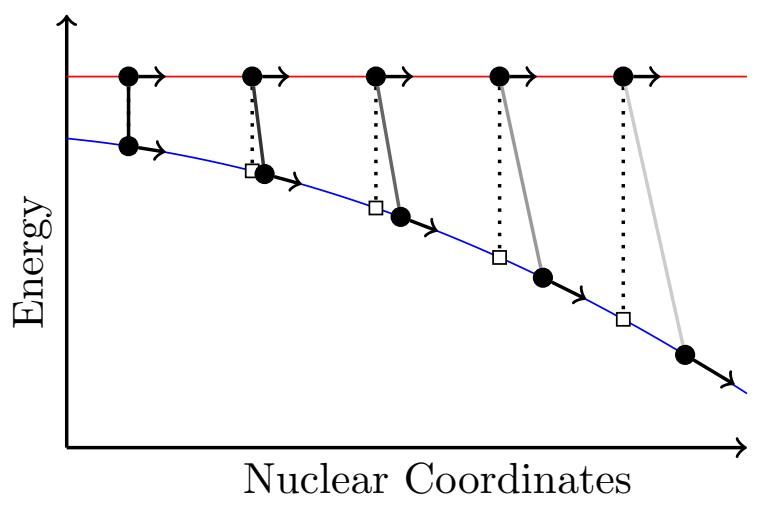

Figure 3: Depiction of an electronic wavepacket propagating on two coupled potential energy surfaces. The solid circles represent the true behaviour of the system: the part of the wavepacket on the lower surface moves faster than the part on the upper surface and therefore the two branches of the wavepacket lose coherence. The empty squares on $S_{1}$ represent the artificially overcoherent state present in surface hopping dynamics and illustrate the need for applying a decoherence correction.

by $c_{\alpha} e^{-\Delta t / \tau_{\alpha \lambda}}$, where $\Delta t$ is the length of a time step, and the coefficient of the active state is then rescaled such that its phase is kept and the total population of all states is 1 .

In the simplified version ${ }^{23}$ of the AFSSH formalism the decoherence rate is computed as

$$
\begin{aligned}
\frac{1}{\tau_{\alpha \lambda}}=\frac{\left(\mathbf{F}_{\alpha \alpha}-\mathbf{F}_{\lambda \lambda}\right) \cdot\left(\delta \mathbf{R}_{\alpha}-\delta \mathbf{R}_{\lambda}\right)}{2 \hbar}- \\
\frac{2\left|\mathbf{F}_{\alpha \lambda} \cdot\left(\delta \mathbf{R}_{\alpha}-\delta \mathbf{R}_{\lambda}\right)\right|}{\hbar}
\end{aligned}
$$

with $\mathbf{F}_{\alpha \lambda}$ defined as $-\left\langle\Psi_{\alpha}|\nabla \hat{\mathrm{H}}| \Psi_{\lambda}\right\rangle$ where $\Psi_{\alpha}$ and $\Psi_{\lambda}$ are electronic wavefunctions in the diagonal representation, $\hat{\mathrm{H}}$ is the $\mathrm{MCH}$, and $\nabla$ refers to nuclear displacements. $\delta \mathbf{R}_{\alpha}$ is the position of the auxiliary trajectory belonging to state $\alpha$. These auxiliary trajectories are propagated in a diabatic picture using a force that is proportional to the state population $\left|c_{\alpha}\right|^{2}$ as described in Ref. 23. The second term in Eq. (7) requires the evaluation of nonadiabatic coupling terms at every time step. In order to lift this requirement, we project the term onto the nuclear velocity $\mathbf{v}$ and discretise the derivative to 
obtain

$$
\frac{2\left|H_{\alpha \lambda} \times \mathbf{v} \cdot\left(\delta \mathbf{R}_{\alpha}-\delta \mathbf{R}_{\lambda}\right)\right|}{\Delta t \times \hbar|\mathbf{v}|^{2}}
$$

Here, $H_{\alpha \lambda}$ is an element of the locally diabatic Hamiltonian $^{26}$ that is already used for wavefunction propagation in SHARC. ${ }^{11}$ Note that Eq. (8) is slightly modified with respect to Ref. 23.

AFSSH proceeds by computing the decoherence times for every inactive state and collapsing its amplitude to zero according to a stochastic algorithm. ${ }^{23}$ In addition, two ad hoc criteria are introduced to cause a reset of the auxiliary trajectories without decoherence: (i) after every surface hop and (ii) according to a reset-time derived from the first term of Eq. (7). ${ }^{23}$

As final point, it is important to realise that these decoherence corrections only help to describe how the wavepacket divides into independent branches. Should these branches meet again later, there is no way to describe their interference correctly with independent trajectories.

\subsection{Momentum rescaling and frustrated hops}

A surface hopping algorithm ultimately has to describe the post Born-Oppenheimer exchange of energy between nuclear and electronic degrees of freedom. Practically, this occurs through the momentum rescaling process that is associated with surface hops. A number of different strategies have been devised ${ }^{14,16,36}$ to this purpose. Here, we will consider four of these possibilities, categorised according to which quantity is conserved in the hop. ${ }^{36}$ It is possible to impose conservation of the total energy $E$, conservation of the momenta of all the nuclei $\mathbf{p}$, or both. Further, if both quantities are conserved, then one has to allow at least one degree of freedom where the momentum can change; this can be done along the nonadiabatic coupling (NAC) vector $\mathbf{h}_{\alpha \lambda}=\mathbf{F}_{\alpha \lambda} /\left(E_{\alpha}-E_{\lambda}\right)$ or the gradient difference vector $\mathbf{g}_{\alpha \lambda}=\mathbf{F}_{\alpha \alpha}-\mathbf{F}_{\lambda \lambda}$. The four different momentum rescaling schemes are summarised in Table 1 . In the $E$ scheme the energy is conserved and the full momentum vector is rescaled without changing its direction. In the $\mathbf{p}$ scheme, the momentum is conserved, which means that no rescaling is performed at all. In the $E \mathbf{p}_{\mathbf{h}}$ and $E \mathbf{p}_{\mathbf{g}}$ schemes, the momentum is rescaled along the $\mathbf{h}_{\alpha \lambda}$ and $\mathbf{g}_{\alpha \lambda}$ directions, respectively.

Table 1: Methods for momentum rescaling investigated in this work.

\begin{tabular}{l|l|l} 
& Conserved quantity & $\begin{array}{l}\text { Rescaling } \\
\text { along }\end{array}$ \\
\hline$E$ & Energy & Momentum \\
$\mathbf{p}$ & Momentum & None \\
$E \mathbf{p}_{\mathbf{h}}$ & Energy and momentum & NAC \\
$E \mathbf{p}_{\mathbf{g}}$ & Energy and momentum & Grad. diff.
\end{tabular}

Based on formal arguments ${ }^{9,66,67}$ and numerical results on simple few-state model systems, ${ }^{32,68}$ a number of authors have concluded that $E \mathbf{p}_{\mathbf{h}}$ is the most rigorous option. Here we shall investigate whether this conclusion holds for a larger system containing more states and many degrees of freedom and how strong the effect is. From a practical viewpoint it is worth noting that $E \mathbf{p}_{\mathbf{h}}$ is the only protocol that requires the availability of nonadiabatic coupling vectors, which are not as readily available as energies and gradients are.

Cases, where a surface hop should occur according to the electronic Schrödinger equation but momentum rescaling is not possible, are termed frustrated hops. The $\mathbf{p}$ protocol does not involve any redistribution of energy and, therefore, no frustrated hops are encountered. In the case of the $E$ protocol one only has to assure that there is enough overall kinetic energy in the system and frustrated hops are rare for a large system as considered here. In the case of the Ep protocols one does not only require conservation of energy but also conservation of the momentum vector of the atoms in the system except for a single degree of freedom. As a consequence, two types of frustrated hops occur for the Ep protocols: ${ }^{36}$ (i) energyforbidden hops, which would also be forbidden in the $E$ protocol, and (ii) linear-momentumforbidden hops where there is enough overall 
energy but not enough along the vector used for rescaling ( $\mathbf{h}$ or $\mathbf{g}$ ). Note that the maximal hopping energy in the $E \mathbf{p}$ protocols corresponds to the kinetic energy of one vibrational degree of freedom, which is on average given as $k T / 2$ where $k$ is the Boltzmann constant and $T$ is an effective temperature. It is through this mechanism that the thermal energy $k T$ enters into the surface hopping simulations and that an equilibrium population according to the Boltzmann distribution can be reached. In other words, these frustrated hops are essential in assuring quantum detailed balance, i.e., the statistical ratio of up and down transitions between different energy surfaces. ${ }^{32,33,68}$ It is also interesting to point out that in the $E$ method, the maximal hopping energy depends on an extensive quantity, the kinetic energy, while it depends on an intensive quantity, the temperature, for the $E \mathbf{p}$ methods.

In the case of a frustrated hop the active state does not change. There are two options about what to do with the momentum and these options will be denoted + and - . In the + method the momentum is left unaltered, i.e. nothing at all happens after a frustrated hop. In the method a portion of the momentum is reflected, which effectively means that the trajectory obtains a second chance to pass through the coupling region and hop to the upper surface.

Specifically, we reflect the momentum parallel to the $\mathbf{h}$ or $\mathbf{g}$ vectors for the $E \mathbf{p}_{\mathbf{h}}$ and $E \mathbf{p}_{\mathbf{g}}$ methods, respectively. Following Refs 23 and 24 this reversion is only done if the following two conditions are fulfilled:

$$
\begin{array}{r}
\left(\mathbf{F}_{\lambda \lambda} \cdot \mathbf{f}_{\lambda \alpha}\right)\left(\mathbf{F}_{\alpha \alpha} \cdot \mathbf{f}_{\lambda \alpha}\right) \\
\left(\mathbf{F}_{\alpha \alpha} \cdot \mathbf{f}_{\lambda \alpha}\right)\left(\mathbf{p} \cdot \mathbf{f}_{\lambda \alpha}\right)<0
\end{array}
$$

Here $\lambda$ is the current active state of the dynamics and $\alpha$ is the state the trajectory would have reached through the frustrated hop; $\mathbf{f}_{\lambda \alpha}$ refers to $\mathbf{h}_{\lambda \alpha}$ or $\mathbf{g}_{\lambda \alpha}$ for the $E \mathbf{p}_{\mathbf{h}}$ and $E \mathbf{p}_{\mathbf{g}}$ methods, respectively. The reversal proceeds by reversing the velocity for the atoms individually, thus conserving $|\mathbf{p}|$ and $E$ in the frustrated hop.

We denote the resulting protocols as $E \mathbf{p}_{\mathbf{h}}^{+}$, $E \mathbf{p}_{\mathbf{h}}^{-}, E \mathbf{p}_{\mathbf{g}}^{+}$, and $E \mathbf{p}_{\mathbf{g}}^{-}$. In the case of $E$, it would in principle also be possible to apply the - pro- tocol but we only evaluate + here (i.e. $E$ implicitly means $E^{+}$) for two reasons. First, the number of frustrated hops is small anyway. Second, an application of the $E^{-}$method would imply to revert the full momentum, which means that the whole trajectory simply proceeds in reverse after the frustrated hop which is an unrealistic scenario.

\subsection{Interpretation and analysis}

There is no unique way of obtaining electronic properties from surface hopping simulations considering that one could base the analysis either on the active states of the individual trajectories or on their quantum amplitudes. This problem becomes particularly apparent when attempting to reconstruct a diabatic picture as in the present work but plays a role in almost every case when analysing dynamics, e.g., when computing intersystem crossing rates. In this work, we follow Ref. 18 and investigate three different methods of analysing the diabatic populations during the dynamics. Method 1 considers only the active surfaces defining the diabatic population of state $n$ as

$$
p_{n}(t)=\frac{1}{N_{\text {traj }}} \sum_{l}^{N_{t r a j}}\left|T_{n, \lambda^{l}(t)}^{l}(t)\right|^{2}
$$

Here, $\mathbf{T}^{l}(t)$ is the diabatic-to-adiabatic transformation matrix for trajectory $l$ at time $t$ and $\lambda^{l}(t)$ is the active diagonal state of trajectory $l$ at time $t$. Method 2, on the other hand, ignores the active state and considers the wavefunction coefficients in the diagonal representation $c_{\alpha}$ instead defining the population as

$$
p_{n}(t)=\frac{1}{N_{\text {traj }}} \sum_{l}^{N_{\text {traj }}}\left|\sum_{\alpha} T_{n, \alpha}^{l}(t) c_{\alpha}(t)\right|^{2}
$$

The third method described in Ref. 18 is an attempt to construct a mixed quantum-classical density using a partial Wigner transform and the final working equation given is a combination of Eq. 11 and the off-diagonal part of 
Eq. 12:

$$
\begin{aligned}
& p_{n}(t)=\frac{1}{N_{t r a j}} \sum_{l}^{N_{t r a j}} \\
& \left(\left|T_{n, \lambda^{l}}^{l}\right|^{2}+\sum_{\alpha<\beta} 2 \operatorname{Re}\left(T_{n \alpha}^{l} c_{\alpha} c_{\beta}^{*} T_{n \beta}^{l *}\right)\right)
\end{aligned}
$$

Note that values obtained using Eq. (11) and Eq. (12) must always lie between 0 and 1 and can, thus, be interpreted as probabilities. Eq. (13) may also lead to negative values and values above 1 and must therefore be seen as a quasiprobability distribution in line with more general properties of Wigner functions. ${ }^{69,70}$

\subsection{Computational Details}

Vibronic coupling parameters are taken from Ref. 54 where electronic structure calculations were performed using the B3LYP functional (as defined by Frisch and coworkers ${ }^{71}$ ) and an all electron triple- $\zeta$ basis set. ${ }^{72}$ The scalar relativistic effects were taken into account within the zeroth-order regular approximation (ZORA). ${ }^{73}$ The vertical transition energies for 2 singlets and 4 triplet states were computed with TDDFT ${ }^{74,75}$ at the same level described above under the Tamm-Damcoff approximation $^{76}$ and the adiabatic local density approximation. The non-equilibrium solvation within the linear-response TD-DFT with a high-frequency dielectric constant of 1.77 for water was used. The SOC effects were introduced according to a simplified relativistic perturbative TD-DFT formalism. ${ }^{77,78}$ These electronic-structure calculations were done with the ADF2013 code. $^{79}$

The model multi-dimensional PES are built from the vibrational normal modes of the singlet electronic ground state. From the 108 normal modes of $\left[\operatorname{Re}(\mathrm{im})(\mathrm{CO})_{3}(\text { phen })\right]^{+}$, 15 were selected as the most important ones involved in the excited state decay starting at $2^{1} \mathrm{MLCT}$, as described in Ref. 53. The resulting 15-mode model Hamiltonian accounts for 12 a' modes at $93,235,439,498,552,637,1174,1336$, $1444,1554,1623,1660 \mathrm{~cm}^{-1}$, and for $3 \mathrm{a}^{\prime \prime}$ modes at 90, 475 and $626 \mathrm{~cm}^{-1}$, which cor- respond mainly to metal-carbonyl modes and vibrations localised on the phenanthroline ligand. ${ }^{53}$ The model parameters corresponding to the excited state energies, SOC values, and intrastate and interstate coupling constants associated to those modes are reported in Ref. 54 . Note that a complex representation is chosen for the SOC matrix. All parameters used are supplied along with the output data via an external repository. ${ }^{80}$

The time-dependent Schrödinger equation for the nuclei was solved by employing the MCTDH method. ${ }^{48-50}$ The multiconfigurational nuclear wave-function is expressed as a linear combination of the Hartree products of the time-dependent basis functions, known as single-particle functions. The wavepacket ansatz adapted to the present non-adiabatic problem corresponds to the multiset formulation. For the full model (2 singlets and 4 triplets), 14 diabatic PESs were considered. The mode combination, number of primitive basis and single particle functions used in the simulations is the same as in Ref. 54 . Harmonic-oscillator basis sets were employed. The initial wavepacket corresponds to the harmonic vibrational ground state of the electronic ground state, promoted at time zero to the $2^{1} \mathrm{MLCT}$ absorbing state. The calculations are done with the Heidelberg MCTDH Package (version 8.4.10). ${ }^{81}$

All surface hopping dynamics simulations were performed in the diagonal representation [Fig. 1 (c)] meaning that the complex SOC matrix is included in the PESs. In the case of complex nonadiabatic coupling vectors between two PESs, we made the approximation of only including the real part for momentum rescaling while we note that a more rigorous strategy for treating complex coupling vectors has recently been developed. ${ }^{82}$ For the full model 14 diagonal PESs were considered. The gradients of these mixed PESs were computed by a transformation using gradients and coupling vectors. ${ }^{11}$ For the analysis, the results were transformed back into the diabatic representation as explained in Ref. 83, summing also over the three triplet components and the results were interpreted using Eqs (11)-(13). A 
nuclear time step of 0.5 fs was chosen following a preliminary analysis that this time step length is sufficient, cf. Figure S1. The trajectories were propagated for $500 \mathrm{fs}$ and an ensemble of 200 trajectories was propagated for each surface hopping protocol. A locally diabatic propagation for the wavefunctions was chosen ${ }^{26}$ using 25 substeps per time step. For the decoherence correction we used the EDC method [Eq. (6), $\mathrm{C}=0.1 \mathrm{H}]$, the simplified AFSSH method, ${ }^{23}$ or no correction. Momentum rescaling was performed according to the four options presented in Tab. 1 and the + and - versions for treating frustrated hops as defined in Section 2.4 were used. All surface hopping simulations were done using the SHARC2.0 package. ${ }^{11,59,61}$

The initial conditions for the surface hopping dynamics were chosen with the goal of most closely mimicking the initial conditions used for MCTDH. Nuclear geometries and velocities were, therefore, sampled according to a Wigner distribution of the zero-point vibrational wavefunction within a harmonic approximation. ${ }^{84}$ The electronic wavefunction was prepared in the diabatic $2^{1}$ MLCT state and the initial active state of the trajectory was the diagonal surface most closely resembling this state. Note that this is a non-standard option within SHARC2.0, requiring manual adjustment of the initial wavefunction coefficients. The advantage of this approach is that it assures that the diabatic population of $2^{1} \mathrm{MLCT}$ at time zero is exactly one when evaluated according to Eq. (12). For completeness we want to point out that an alternative way of creating similar initial conditions would amount to assigning an oscillator strength to the diabatic $2^{1}$ MLCT state and subsequently simulating a $\delta$ pulse excitation. It is not a priori clear, which one of these methods reflects the MCTDH initial conditions more aptly.

The quality of the dynamics was gauged by computing a time-averaged absolute error of the diabatic populations computed with surface hopping against the MCTDH reference, defined as

$$
\epsilon_{t}=\frac{\Delta t}{t_{\max }} \sum_{t=\Delta t}^{t_{\max }} \sum_{n=1}^{N_{s t}}\left|p_{n}(t)-p_{n}^{r e f}(t)\right| .
$$

Here, $p_{n}(t)$ is the diabatic population of state $\alpha$ at time $t$ and $p_{n}^{r e f}(t)$ corresponds to the reference value computed with MCTDH. Unless specified, Eq. (13) was used to compute $p_{n}(t)$. In the case of triplet states, the $p_{\alpha}(t)$ value corresponds to the sum over all three components of the state. The $t_{\max }$ value corresponds to the propagation time, i.e. 500 fs. Accordingly, the maximum possible value for the error is 2 , which would be obtained if there is no coincidence at all between the states populated in the two runs. As an alternative error measure, time constants were obtained by fitting first-order kinetics models to the population data. Errors of the time constants were obtained with the bootstrapping method, ${ }^{85,86}$ using 100 bootstrapping samples for each ensemble and considering geometric error windows.

\section{Results}

We start by discussing the states involved in the dynamics and their main dynamical features, as determined by the MCTDH method. Subsequently, we discuss the correct way of interpreting the dynamics. Finally, we shall evaluate the performance of different surface hopping methods on the full model describing $\left[\operatorname{Re}(\mathrm{im})(\mathrm{CO})_{3}(\text { phen })\right]^{+}$and various simplified models.

In line with previous work on this complex, ${ }^{53,54}$ we will consider two singlet states and four triplet states (i.e., a total of 14 individual spin-orbit coupled states). All but one are of predominant MLCT character with the remaining one of IL character. In Table 2, the vertical excitation energies of these states at the $S_{0}$ equilibrium geometry are presented. Two types of labels are given to account for the MCH (labelled by energy, i.e. $S_{1}, S_{2}, T_{1}-T_{4}$ ) and diabatic (labelled by character, e.g. $2^{1} \mathrm{MLCT}$ for the second singlet MLCT state) representations (cf. Fig. 2). Table 2 shows that the six states occupy a narrow energy range of only $0.5 \mathrm{eV}$, suggesting rapid nonadiabatic transitions.

To simulate the dynamics of the complex after vertical excitation, the system was prepared in the diabatic $2^{1} \mathrm{MLCT}$ state and propagated for 
Table 2: Singlet and triplet vertical excitations of the $\left[\operatorname{Re}(\mathrm{im})(\mathrm{CO})_{3}(\text { phen })\right]^{+}$complex: energies and state labels in the $\mathrm{MCH}$ and diabatic representations.

\begin{tabular}{ccc}
\hline MCH & Diabatic & $\Delta E(\mathrm{eV})$ \\
\hline$S_{1}$ & $1^{1} \mathrm{MLCT}$ & 3.12 \\
$S_{2}$ & $2^{1} \mathrm{MLCT}$ & 3.40 \\
$T_{1}$ & $1^{3} \mathrm{MLCT}$ & 2.98 \\
$T_{2}$ & $2^{3} \mathrm{MLCT}$ & 3.07 \\
$T_{3}$ & $1^{3} \mathrm{IL}$ & 3.24 \\
$T_{4}$ & $3^{3} \mathrm{MLCT}$ & 3.42 \\
\hline
\end{tabular}

500 fs. In Fig. 4 (a) dynamics computed at the MCTDH level of theory is presented. The first process observed is an ultrafast transfer of population from $2^{1} \mathrm{MLCT}$ to the almost quasidegenerate $3^{3} \mathrm{MLCT}$ state mediated via SOC and after only 12 fs the population of $2^{1} \mathrm{MLCT}$ has already decayed to 0.5 . The $3^{3} \mathrm{MLCT}$ state reaches a maximum at only 24 fs and subsequently decays while populating the other triplet states and also transferring back some of the population to $2^{1}$ MLCT. After 500 fs about $59 \%$ of the population is in $1^{3}$ MLCT while the remaining part is about evenly split between $2^{3} \mathrm{MLCT}$ and $1^{3} \mathrm{IL}$.

Figure 4 (b) shows the evolution of the same dynamics obtained with surface hopping and selecting the EDC $/ E \mathbf{p}_{\mathbf{h}}^{-}$option. At this level the agreement between surface hopping and MCTDH is very good and all the features mentioned above are well reproduced. The use of SHARC allows us to easily convert these results into the $\mathrm{MCH}$ representation, which is shown in Fig. 4 (c). The MCH populations closely resemble the diabatic ones with the exception that the lower energy states have larger populations than their corresponding diabatic states; for example, the rise of $T_{4}$ in the early dynamics is somewhat lower than the rise of $3^{3} \mathrm{MLCT}$ and the population of $T_{1}$ at the end of the simulated period is somewhat larger than the population of $1^{3}$ MLCT. This is expected as highenergy diabatic states mix with lower-lying energy states.

We also want to briefly address the question of how much the truncation of the model to 15 modes could affect the overall dynam- ics within the LVC approximation. For this purpose, we recomputed the surface hopping dynamics including all 108 normal modes of $\left[\operatorname{Re}(\mathrm{im})(\mathrm{CO})_{3}(\text { phen })\right]^{+}$and the corresponding linear vibronic coupling constants. The results, determined at the $E D C / E \mathbf{p}_{\mathbf{h}}^{-}$level, are presented in Fig. 4 (d). This figure closely represents the corresponding results for the 15-mode model (Fig. 4 (b)) with only a few exceptions, e.g. the rise of $3^{3} \mathrm{MLCT}$ in the early dynamics is somewhat less pronounced and there is no second rise of $2^{1} \mathrm{MLCT}$. We therefore conclude that the 15-mode model is a reasonable approximation of the overall complex, at least within a harmonic approximation.

Before we can address the main concern of this work, the question of which surface hopping protocol is optimally suited to describe the system at hand, we have to proceed by scrutinising the possibilities of interpreting the populations in the dynamics. For this purpose, we evaluated the three methods described in Ref. 18, which are summarized in Eqs (11)-(13). To show the effects of these methods, we select the $\mathrm{AFSSH} / E \mathbf{p}_{\mathbf{h}}^{-}$method, which turned out to be particularly illustrative for this purpose (see below). The results are shown in Fig. 5 where the major states involved in the dynamics ( $\left.2^{1} \mathrm{MLCT}, 3^{3} \mathrm{MLCT}, 1^{3} \mathrm{MLCT}\right)$ are shown in panel (a) and the secondary states in panel (b). The MCTDH reference and surface hopping results are shown in solid and dotted lines respectively. We use three different markers to denote the three methods of collecting the populations: Method $1(\times)$, Method $2(\triangle)$, and Method $3(\mathrm{o})$. In the left panel, a zoom into the first $30 \mathrm{fs}$ is provided. This reveals the striking observation that the initial conditions are strongly different between the different methods. Only Method $2(\triangle)$, i.e. averaging over the quantum amplitudes, assures that the initial population of $2^{1} \mathrm{MLCT}$ is exactly one, which corresponds to the setting used in the MCTDH population. Simply using the information from the active surface (Method 1, $\times$ ) strongly differs from this picture and only $64 \%$ of the population starts in $2^{1}$ MLCT while the remaining population is distributed over $3^{3} \mathrm{MLCT}$ and $1^{3} \mathrm{IL}$. The reason for this discrep- 

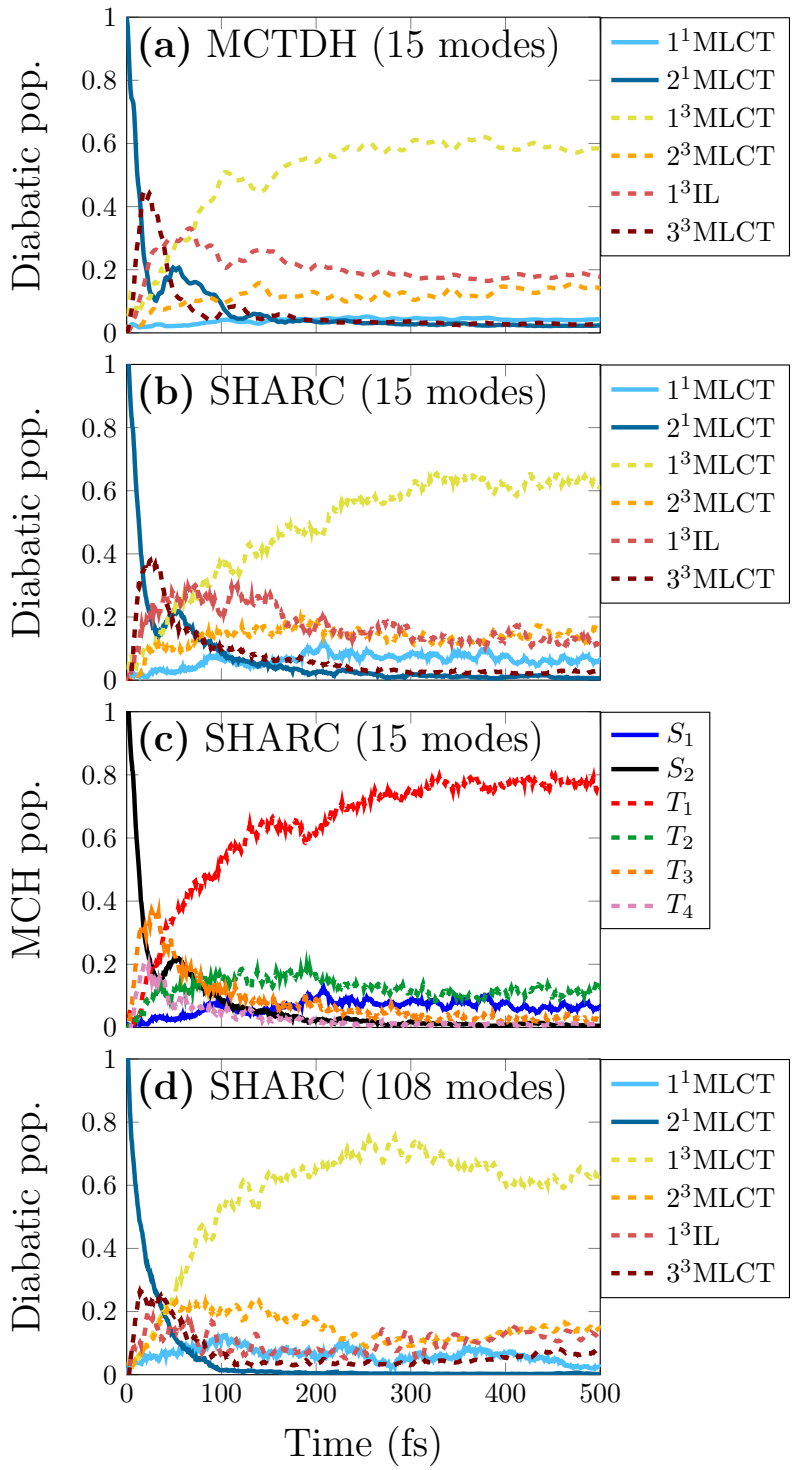

Figure 4: Time-evolution of the state populations of the full model considering (a) diabatic populations at the MCTDH level (15 modes), (b) diabatic populations (15 modes), (c) $\mathrm{MCH}$ populations (15 modes), (d) diabatic populations (all 108 modes) at the surface hopping $\left(\mathrm{EDC} / E \mathbf{p}_{\mathbf{h}}^{-}\right)$level.

ancy is that the diagonal surfaces present at the Franck-Condon geometry are strongly mixed containing contributions from various diabatic states. Therefore, a strongly mixed picture is obtained when simply averaging over the active states according to Eq. (11). Proceeding to Method 3 (०), we find that the initial quasiprobability of $2^{1} \mathrm{MLCT}$ is 1.14 , i.e. even larger than one. This is compensated by slightly negative quasiprobabilities for all the other states.
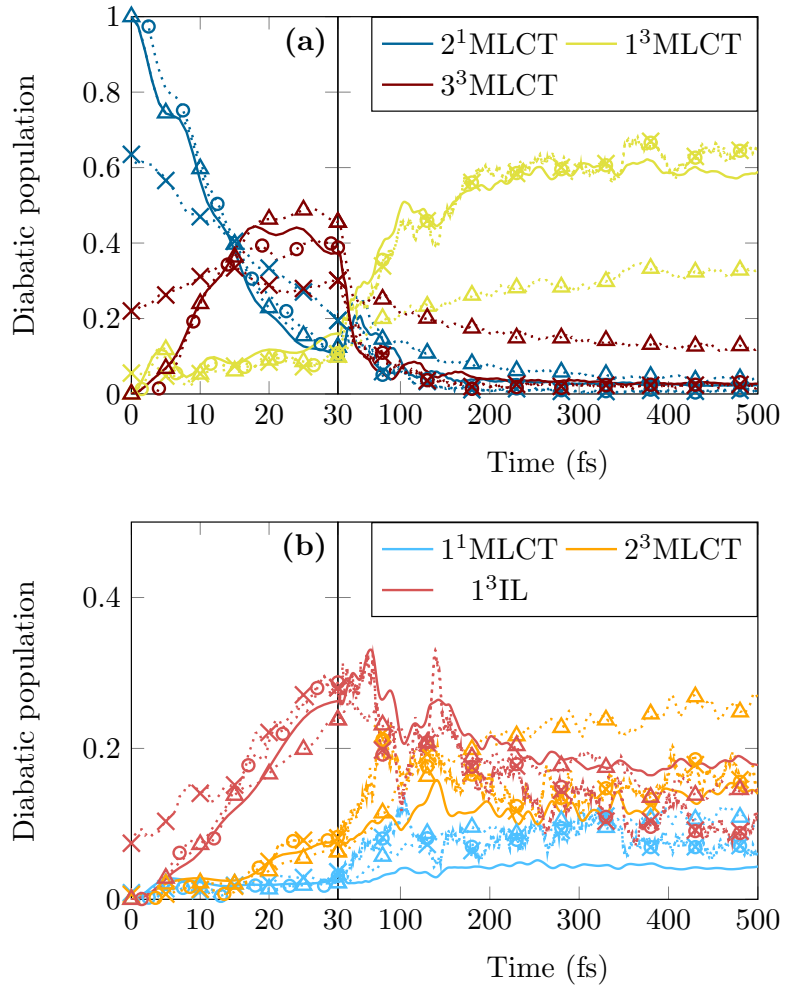

Figure 5: Time-evolution of the diabatic state populations of the full model computed at the MCTDH level (solid lines) compared to the $\mathrm{AFSSH} / E \mathbf{p}_{\mathbf{h}}^{-}$level of theory (dotted lines) using three different methods of defining the populations as described in the text: Method $1(x)$, Method $2(\triangle)$, and Method $3(\circ)$.

Proceeding to the dynamics, we find that Methods $2(\triangle)$ and 3 (o) closely follow the MCTDH dynamics within the first 30 fs. However, these methods diverge later on and Method 2 shows a significantly reduced transfer to the $1^{3} \mathrm{MLCT}$ state when compared to the MCTDH reference. Interestingly, at the later stage of the dynamics Methods $1(x)$ and 3 (o) almost coincide providing good agreement with MCTDH. Two important conclusions follow from this comparison. First, we find that the mixed quantum-classical Method 3 provides good agreement with MCTDH over the full range of the dynamics and we will therefore proceed to use this method for the subsequent analysis. Second, the main deficiency of using the quantum amplitudes (Method 2, $\triangle$ ) is that the population of the higher energy states is overestimated. This can be understood in the following way: The propagation of the electronic 
wavefunction leads to enhanced quantum amplitudes of the higher energy states but these states are not actually populated during the dynamics due to frustrated hops. This finding illustrates that frustrated hops are essential in assuring that the lower energy states are correctly populated.

We are now in the position to address the main concern of this work: How well is the dynamics reproduced by using different approximations within the surface hopping method? For this purpose, we have evaluated the surface hopping dynamics using 13 different levels of theory, where the decoherence correction, the mode of momentum rescaling and the treatment of frustrated hops are varied. The results are summarised in Fig. 6, see also the individual results in Figures S2-S14 of the supporting information. The bars of Fig. 6 are computed as floating averages on a logarithmic time scale considering the following intervals $0-1 \mathrm{fs}, 1-2$ fs, 2-4 fs, 4-8 fs until 256-500 fs, and coloured according to their electronic and spin character. The upper left panel displays compactly the MCTDH results from Fig. 4 (a). Here, one can see the initial population of $2^{1} \mathrm{MLCT}$, which is the dominant state until the fifth bar (the interval 8-16 fs). Then, the $3^{3} \mathrm{MLCT}$ state dominates (16-32 fs) while in the final interval more than $50 \%$ of the population is in the $1^{3} \mathrm{MLCT}$ state.

Gratefully, all the methods considered show an appropriate time scale for the $2^{1} \mathrm{MLCT}$ decay and correctly predict the intermediate rise of $3^{3}$ MLCT. However, half of them fall short when describing the outcome at the end of the dynamics and only the $E \mathbf{p}$ protocols, shown on the right side, correctly place at least about $50 \%$ of the population in the $1^{3} \mathrm{MLCT}$ state. To provide a more quantitative discussion of the deviations we compute the mean-absolute error $\epsilon$ [Eq. (14)]. The two best-performing methods are $\mathrm{EDC} / E \mathbf{p}_{\mathbf{g}}^{-}$and $\mathrm{EDC} / E \mathbf{p}_{\mathbf{h}}^{-}$closely followed by their AFSSH counterparts. The various + protocols follow, all giving an error less or equal to 0.25 . The different $E$ and $\mathbf{p}$ methods, shown on the left side, exhibit significantly enhanced errors. Although a detailed discussion will be done in the next section, we emphasise now that the errors introduced by the different surface hopping algorithms are non-negligible and that supposedly unimportant algorithmic details do affect the results strongly.

Noting the challenges involved in the full model of $\left[\operatorname{Re}(\mathrm{im})(\mathrm{CO})_{3}(\text { phen })\right]^{+}$, where the interactions of 14 spin-orbit coupled states have to be modelled correctly, we want now to get a deeper insight into the effect of the different protocols by studying simpler models. For this purpose, we use the same set of parameterised 15 normal modes but a smaller number of states. First, we consider only the two singlet states $1^{1} \mathrm{MCLT}$ and $2^{1} \mathrm{MCLT}$. When using MCTDH (upper left panel of Fig. 7), the interconversion between these two states happens on the time scale of a few hundred femtoseconds and for the last two bars, i.e. the time after $128 \mathrm{fs}$, more than $50 \%$ of the population is in $1^{1}$ MCLT. It can be readily seen that the interconversion is too slow with all surface hopping protocols as none of them has two bars below the line indicating a population of 0.5. Interestingly, the EDC protocols have significantly enhanced error bars when compared to their AFSSH counterparts. For this model, the AFSSH $/ E \mathbf{p}_{\mathbf{h}}^{-}$method gives the best performance giving only a slightly slower decay than MCTDH.

We systematically increase the complexity of the model, considering the interaction between the $2^{1} \mathrm{MCLT}$ and $1^{3} \mathrm{IL}$ states that gives rise to four spin-orbit coupled states. The upper left panel of Fig. 8 shows that intersystem crossing should occur on a similar time scale to the internal conversion of the previous case. However, the underlying physics is clearly different as $2^{1} \mathrm{MCLT}$ and $1^{3} \mathrm{IL}$ are coupled via SOC whereas the singlet states in Fig. 7 are connected by non-relativistic vibronic coupling. In addition, the position of the minimum of the $1^{3} \mathrm{IL}$ potential surface is significantly altered when compared to the MLCT states. ${ }^{53}$ As a consequence of these changes in parameters, strongly varying outcomes are observed for the different surface hopping methods. In general, improved results are obtained with respect to the previous case and most methods show acceptable error bars. Some trends are visible: 

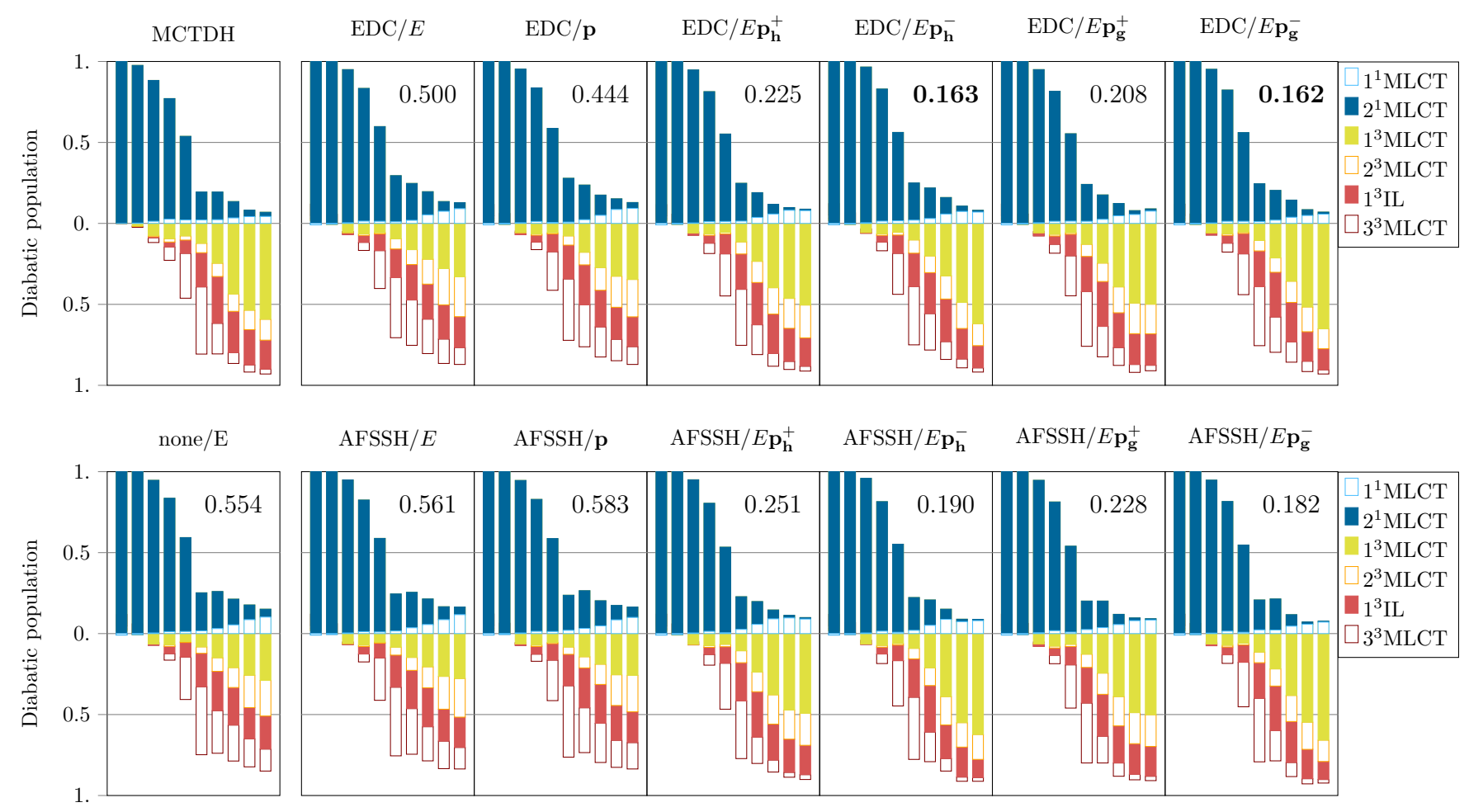

Figure 6: Diabatic electronic populations (using Eq. (13)) for the full model of $\left[\operatorname{Re}(\mathrm{im})(\mathrm{CO})_{3}(\text { phen })\right]^{+}$plotted on a logarithmic time scale for 0-500 fs after photoexcitation considering different surface hopping protocols. The numbers printed are the mean-absolute errors with the best performers marked in bold.

the - protocols generally outperform the + protocols and EDC outperforms AFSSH. Interestingly, the best performer is the EDC/p method, meaning that no momentum rescaling is performed at all after a hop. It may also be noted that the $2^{1} \mathrm{MCLT} / 1^{3} \mathrm{IL}$ model includes no interstate vibronic coupling constants but only SOC. In the current implementation this yields a situation where the $E \mathbf{p}_{\mathbf{h}}$ and $E \mathbf{p}_{\mathbf{g}}$ protocols yield exactly identical outcomes.

Finally, we consider the $3^{3} \mathrm{MLCT}$ and $1^{3} \mathrm{IL}$ states, which give rise to 9 states interacting via SOC and vibronic coupling, see Fig. 9. In this case the interconversion occurs somewhat faster than in the previous cases and after 42 fs half the population is already in the $1^{3} \mathrm{IL}$ state according to the MCTDH reference. Most of the surface hopping protocols perform quite well in this case with $\epsilon<0.15$; only $\mathrm{EDC} / E$ and $\mathrm{EDC} / \mathbf{p}$ are above this threshold. The best performing method is $\mathrm{AFSSH} / E \mathbf{p}_{\mathbf{g}}^{-}$.

A more compact form of the results is presented in Table 3, where the mean absolute errors are averaged over the four types of dynamics considering the three different methods of obtaining diabatic populations described in Eqs (11)-(13). In the case of Methods 1 and 3 , the best performer is $\mathrm{AFSSH} / E \mathbf{p}_{\mathbf{h}}^{-}$, an implementation that closely follows Ref. 23. For Method 2, the best performer is $\mathrm{EDC} / E \mathbf{p}_{\mathbf{h}}^{-}$. More generally, it is observed that $E \mathbf{p}_{\mathbf{h}}^{-}$, i.e. momentum rescaling along the coupling vector and reflection of frustrated hops, tends to be the best performer in its respective row. This result is closely followed by $E \mathbf{p}_{\mathbf{g}}^{-}$. This is followed by the different + methods and finally the $E$ and $\mathbf{p}$ methods. In terms of the decoherence corrections we do not find a clear preference between EDC and AFSSH but it can be seen that the EDC and AFSSH values are better than not performing any correction at all. By far the largest error in all of Table 3 is obtained for none/ $E$ when interpreted according to Method 2. In other words: If one chooses to run dynamics without any decoherence correction, it is imperative to include information about the 


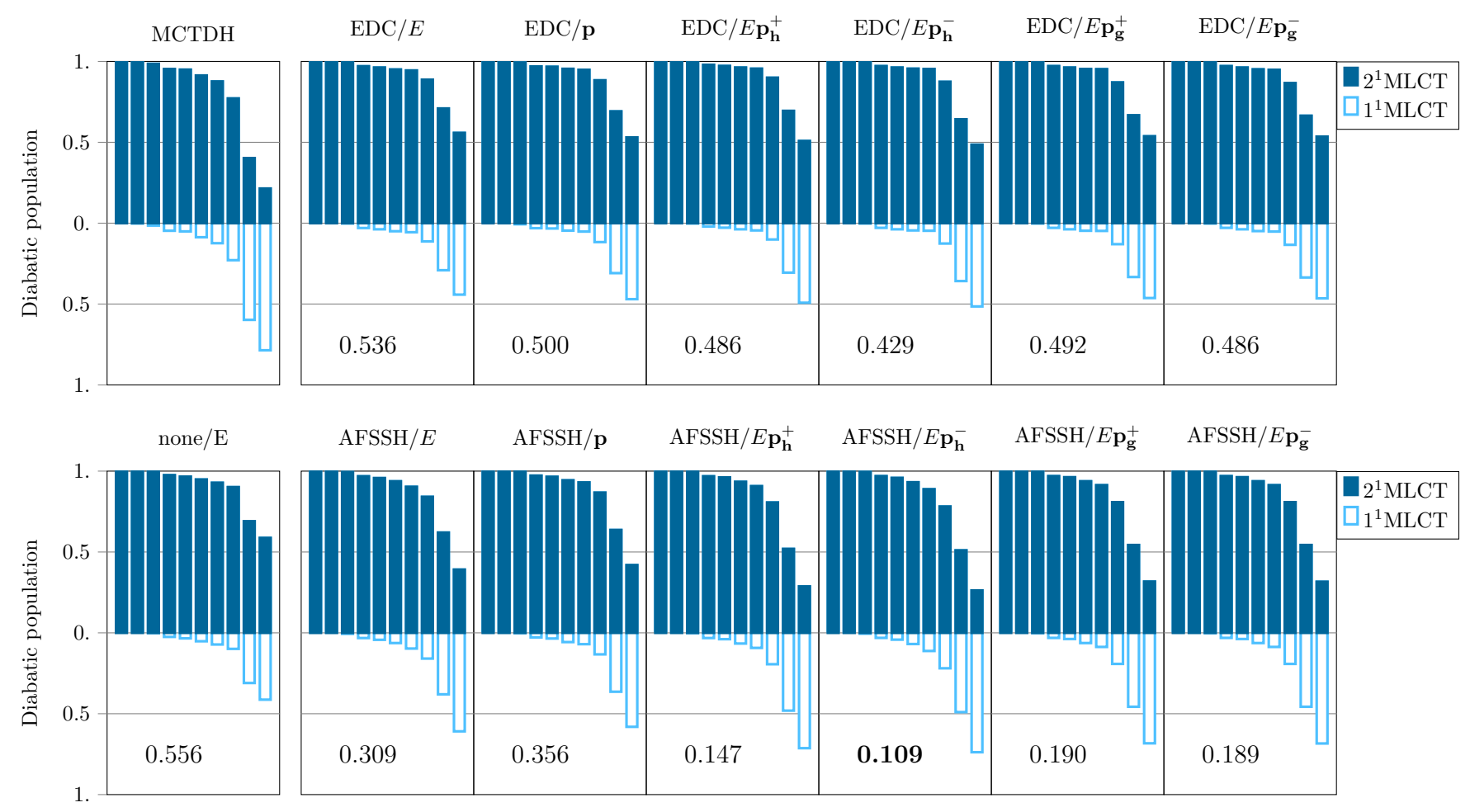

Figure 7: Diabatic electronic populations (using Eq. (13)) for the singlet MCLT states plotted on a logarithmic time scale for 0-500 fs after photoexcitation considering different surface hopping protocols. The numbers printed are the mean-absolute errors with the best performer marked in bold.

active surfaces of the trajectories rather than analysing only the quantum amplitudes.

A complementary perspective of the dynamics can be achieved by fitting time constants. For this purpose, the following kinetic models were considered

$$
\begin{aligned}
S_{2}+S_{1} & \rightleftharpoons T_{4}+T_{3}+T_{2}+T_{1} \\
S_{2} & \rightarrow S_{1} \\
S_{2} & \rightarrow T_{3} \\
T_{4} & \rightleftharpoons T_{3}
\end{aligned}
$$

and the data fitted appropriately. In all these cases, only the decay times for the forward reaction were compared for simplicity although for Eq. (15) and Eq. (18) we also fitted the backwards reaction rate. The obtained times are plotted in Fig. 10 on a logarithmic scale. The dotted lines indicate the reference values computed at the MCTDH level and the shaded areas correspond to logarithmic error values between $3 / 4$ and $4 / 3$ times the reference time. In the case of the full model, we fitted the overall intersystem crossing as a reversible interconversion between singlets and triplets [cf. Eq. (15)]. The reference time for the forward intersystem crossing was determined as $16.0 \mathrm{fs}$. All the surface hopping simulations stay well within the indicated error window and give time constants that are only slightly larger. The $2^{1} \mathrm{MLCT} / 1^{1} \mathrm{MLCT}$ system decays with a time constant of 235 fs for MCTDH. Interestingly, all the surface hopping protocols overshoot this value and only the $\mathrm{AFSSH} / E \mathbf{p}_{\mathbf{h}}^{-}$and $\mathrm{AFSSH} / E \mathbf{p}_{\mathbf{h}}^{+}$protocols stay within the specified error window. The $2^{1} \mathrm{MLCT} / 1^{3} \mathrm{IL}$ interconversion occurs on a very similar time scale to the previous case at the MCTDH level (239 fs). As opposed to the previous case, most of the employed surface hopping methods underestimate this time and four methods are within the error window: $\mathrm{EDC} / E, \mathrm{EDC} / \mathbf{p}, \mathrm{EDC} / E \mathbf{p}_{\mathbf{h}}^{-}$, and $E D C / E \mathbf{p}_{\mathbf{g}}^{-}$. The interconversion between $3^{3} \mathrm{MLCT}$ and $1^{3} \mathrm{IL}$ occurs with a time constant of 57 fs at the MCTDH level. This time constant is in most cases overestimated by surface 


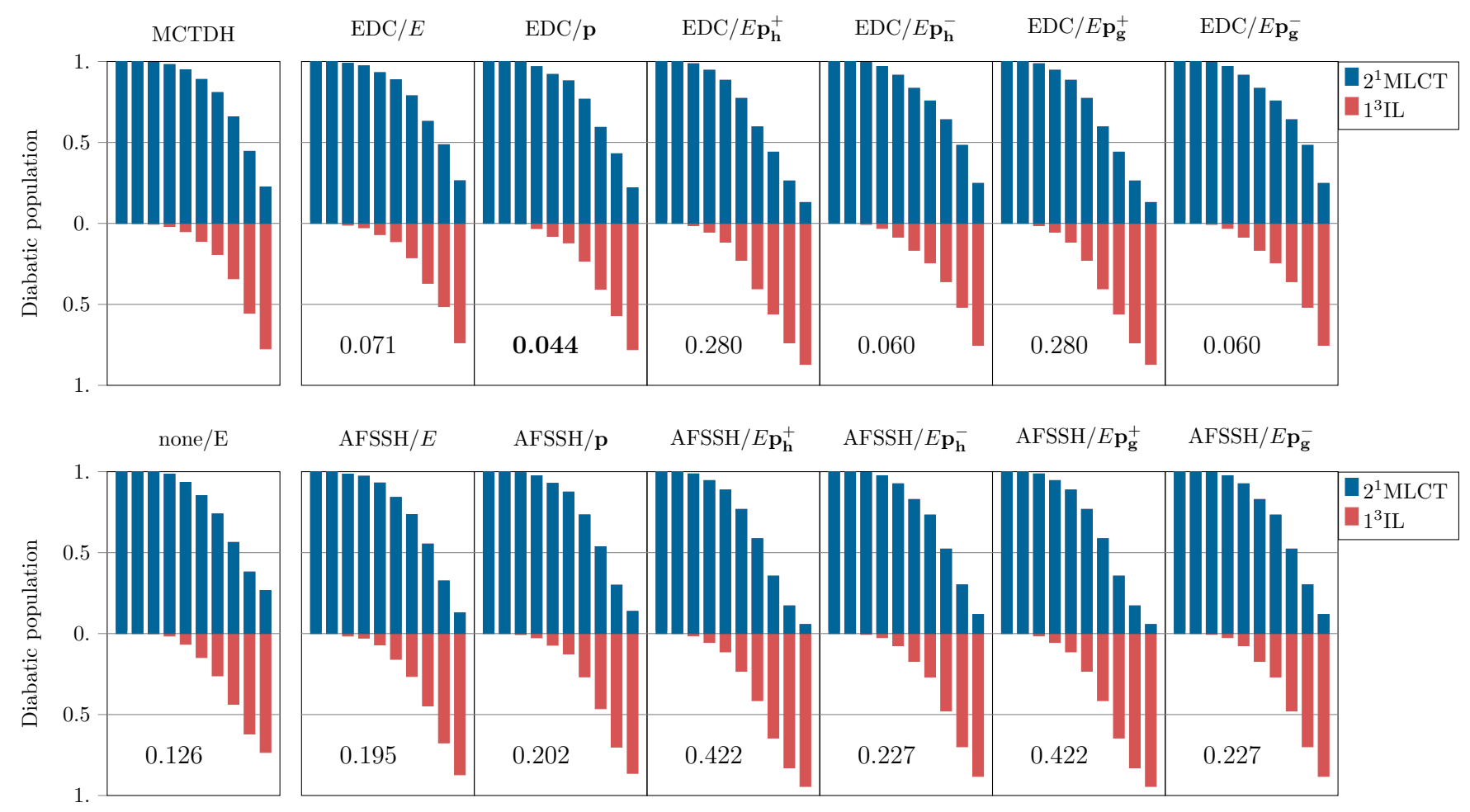

Figure 8: Diabatic electronic populations (using Eq. (13)) for the $2^{1}$ MLCT and $1^{3}$ IL states plotted on a logarithmic time scale for 0-500 fs after photoexcitation considering different surface hopping protocols. The numbers printed are the mean-absolute errors with the best performer marked in bold.

hopping but six methods stay within the specified error window.

Figure 10 evidences that the evaluated surface hopping protocols do reproduce the correct order of magnitude but none of them places all four time constants within the given error window. However, the $\mathrm{AFSSH} / E \mathbf{p}_{\mathbf{h}}^{+}$and $\mathrm{AFSSH} / E \mathbf{p}_{\mathbf{h}}^{-}$keep at least three out of the four time constants within the specified window. Moreover, one sees that the errors are generally not uniform as the time scales are sometimes overestimated and sometimes underestimated, showing that there is probably no simple solution. An interesting trend is the fact that all the + methods decay on shorter time scales than the - methods. This may be understood in the following simple picture: The - protocol redirects the trajectory into the crossing region after a frustrated hop. Thus, the trajectory obtains a second chance to hop up into the higher state, which leads to the fact that the overall decay to the lower state is slowed down.

It is also of interest to discuss whether the results are statistically significant, i.e. whether 200 trajectories are enough to provide almost converged decay times. This is assessed using a bootstrap algorithm ${ }^{85,86}$ that estimates the error related to the finite number of trajectories. The results are presented as error bars in Fig. 10. As these errors are significantly smaller than the fluctuations between the different methods, it is fair to assume that none of the conclusions would change if a larger number of trajectories were run.

Finally, it is of interest to assess whether the time constants change if a different method for computing the diabatic populations is used. For this purpose, we have recomputed the decay times using Eq. (12), see Figure S54. It is worth pointing out that the time-constants have a non-negligible dependence on this. The results are particularly dramatic in the case of the none/E protocol where Eq. (13) gives decay times of $17 / 667 / 227 / 90$ fs for the four models while these values are 14/970/570/108 fs in the case of Eq. (12). This illustrates that the 

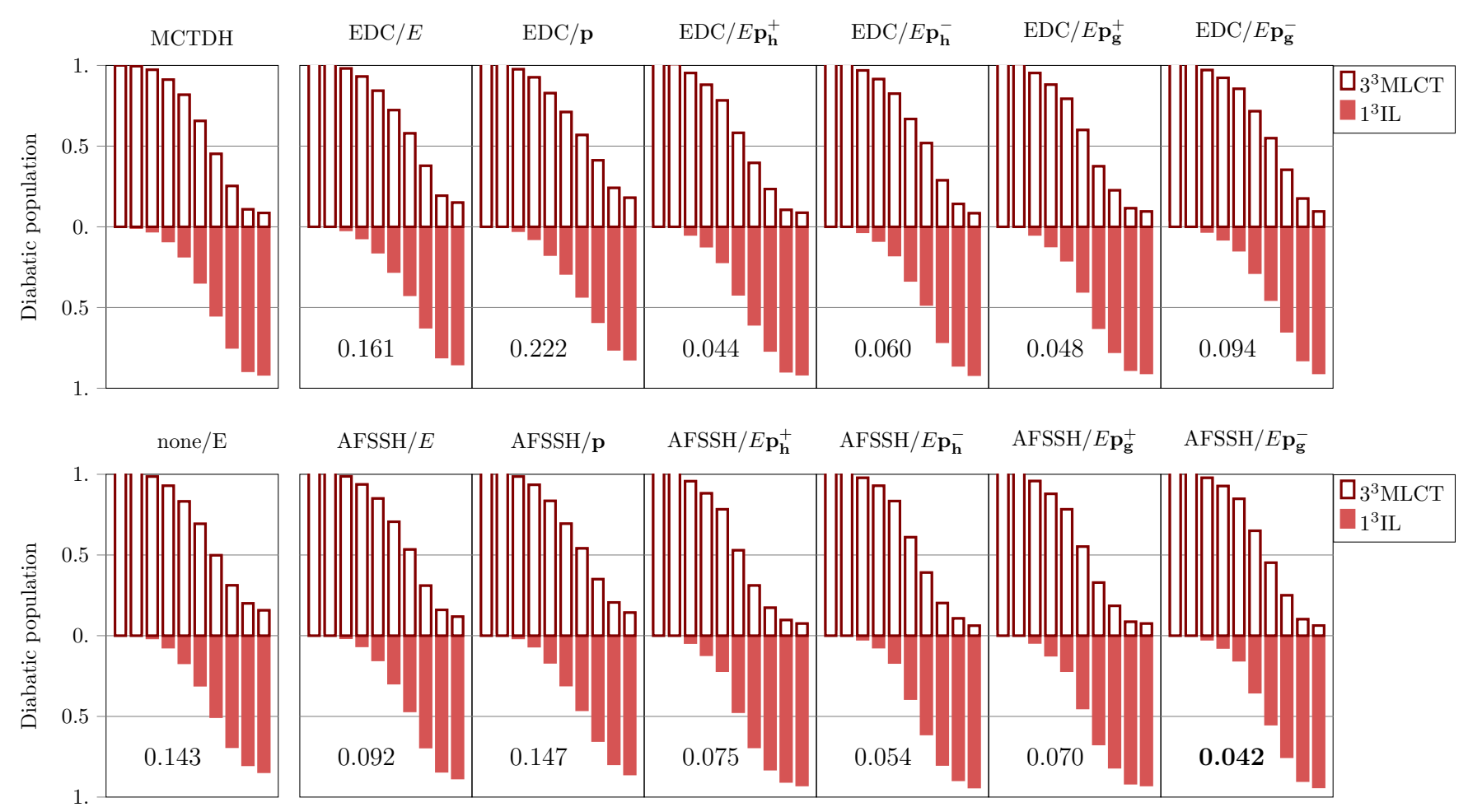

Figure 9: Diabatic electronic populations (using Eq. (13)) for the $3^{3}$ MLCT and $1^{3} \mathrm{IL}$ states plotted on a logarithmic time scale for 0-500 fs after photoexcitation considering different surface hopping protocols. The numbers printed are the mean-absolute errors with the best performer marked in bold.

method of analysing the populations does not only affect the more abstract error measures discussed above but also has a very tangible effect in terms of affecting the fitted decay times.

\section{Discussion}

The above results illustrate that algorithmic details within surface hopping can have a strong impact, while also showing that for all four models there is at least one protocol that yields good results. Whereas it is difficult to draw a final conclusion, a number of clear trends emerge. For all the models considered and ways to evaluate the results, it is found that momentum rescaling along the nonadiabatic coupling vector $\left(E \mathbf{p}_{\mathbf{h}}\right)$ is superior to the three other evaluated methods. It has been argued previously that this is the most rigorous way of momentum rescaling. 9,66,67 The main effect seen by the application of $E \mathbf{p}_{\mathbf{h}}$ is an enhanced number of frustrated hops, which leads to the fact that the trajectory correctly remains trapped in the lower state. This is similar to the results of Refs 32,33,68 which discuss this effect in terms of detailed balance. This effect is particularly drastic whenever a large number of states are present in a narrow energy window as is the case for transition metal complexes. The challenge of applying the $E \mathbf{p}_{\mathbf{h}}$ protocol is that it requires the availability of nonadiabatic couplings, which are not straightforward to calculate with most of the electronic structure codes available. With this hurdle in mind, we have attempted the same protocol only replacing the nonadiabatic coupling with the gradient difference $\left(E \mathbf{p}_{\mathbf{g}}\right)$. We find that this approach performs almost as well as the more rigorous $E \mathbf{p}_{\mathbf{h}}$ method. This suggests that the most important feature here is to limit the energy that is available for upward hops. Both protocols restrict the maximum energy for upward hops to the energy of one degree of freedom (i.e. $k T / 2$ at thermal equilibrium). Following this line of argument suggests that one may get away with a more pragmatic approach such as restrict- 
Table 3: Mean absolute errors averaged over the four types of trajectories computed for three different methods of obtaining the populations.

\begin{tabular}{|c|c|c|c|c|c|c|c|}
\hline & & $E$ & p & $E \mathbf{p}_{\mathbf{h}}^{+}$ & $E \mathbf{p}_{\mathbf{h}}^{-}$ & $E \mathbf{p}_{\mathrm{g}}^{+}$ & $E \mathbf{p}_{\mathrm{g}}^{-}$ \\
\hline Method $1^{\mathrm{a}}$ & $\mathrm{EDC}$ & 0.315 & 0.307 & 0.273 & 0.182 & 0.271 & 0.203 \\
\hline \multirow[t]{2}{*}{ Classical } & AFSSH & 0.296 & 0.328 & 0.231 & 0.156 & 0.235 & 0.171 \\
\hline & none & 0.350 & & & & & \\
\hline Method $2^{b}$ & $\mathrm{EDC}$ & 0.306 & 0.298 & 0.264 & 0.177 & 0.262 & 0.193 \\
\hline \multirow[t]{2}{*}{ Quantum } & AFSSH & 0.313 & 0.343 & 0.302 & 0.242 & 0.302 & 0.239 \\
\hline & none & 0.616 & & & & & \\
\hline Method $3^{\mathrm{c}}$ & EDC & 0.317 & 0.302 & 0.259 & 0.178 & 0.257 & 0.200 \\
\hline \multirow[t]{2}{*}{ Mixed } & AFSSH & 0.289 & 0.322 & 0.224 & 0.145 & 0.227 & 0.160 \\
\hline & none & 0.345 & & & & & \\
\hline
\end{tabular}

ing the maximal hopping energy to a value of $E_{k i n} / N_{d o f}$ where $N_{d o f}$ is the number of degrees of freedom or to a value of $k T / 2$ with a predefined temperature $T$. Note that these choices yield a size-intensive ${ }^{87}$ (but not necessarily sizeconsistent) maximal hopping energy meaning that it does not grow arbitrarily large as the system size increases.

Considering that the number of frustrated hops is the main feature that sets the Ep methods apart from the others, we have evaluated different options of what to do after a frustrated hop in these two cases and found that reflection $(-)$ generally outperforms completely ignoring the hops $(+)$. The - protocol reflects the trajectory back into the crossing region and provides a second chance of hopping into the higher state. As a consequence, we find that the net transfer to the lower state is slowed down whenever the - protocol is used. As discussed above, the - protocol does not make much sense for simple rescaling along the momentum $\left(E^{-}\right)$ and was not investigated here.

Unfortunately, the question of decoherence correction does not have a straightforward answer. While our results emphasise the importance of a decoherence correction, they also show that none of the EDC and AFSSH protocols provided completely satisfactory solutions. The problem of these methods may be understood in the sense that neither of them is sizeconsistent in the present implementation. The
EDC method [Eq. (6)] depends on the overall kinetic energy $E_{k i n}$ of the system. Adding noninteracting atoms to the system will increase the overall kinetic energy and thus reduce the decoherence time. In the case of AFSSH the argument is more subtle. Following Ref. 23, the positions and momenta of the auxiliary trajectories are reset after every surface hop. In the full model of the complex considered there are 14 states present within as little as $0.5 \mathrm{eV}$. As a consequence, an exorbitant number of hops occur, mostly related to trivial crossings, meaning that the auxiliary trajectories never have the time to properly build up to induce decoherence and as a consequence the decoherence rate is strongly underestimated. More generally, we can say that the AFSSH algorithm is not sizeconsistent with respect to the density of states in the system as the addition of non-interacting electronic states to any model system will lower the decoherence rate through an enhanced number of surface hops. The shortcomings of both methods, EDC and AFSSH, may be addressed in the future. In the case of EDC one may enforce size-intensivity by restricting the decoherence to the kinetic energy of one designated degree of freedom as in the original formulation $^{20}$ or by simply replacing the $E_{k i n}$ term of Eq. (6) by $E_{k i n} / N_{d o f}$. The problem of AFSSH might be solved by using a more sophisticated implementation of the AFSSH algorithm that treats trivial crossings differently from true 


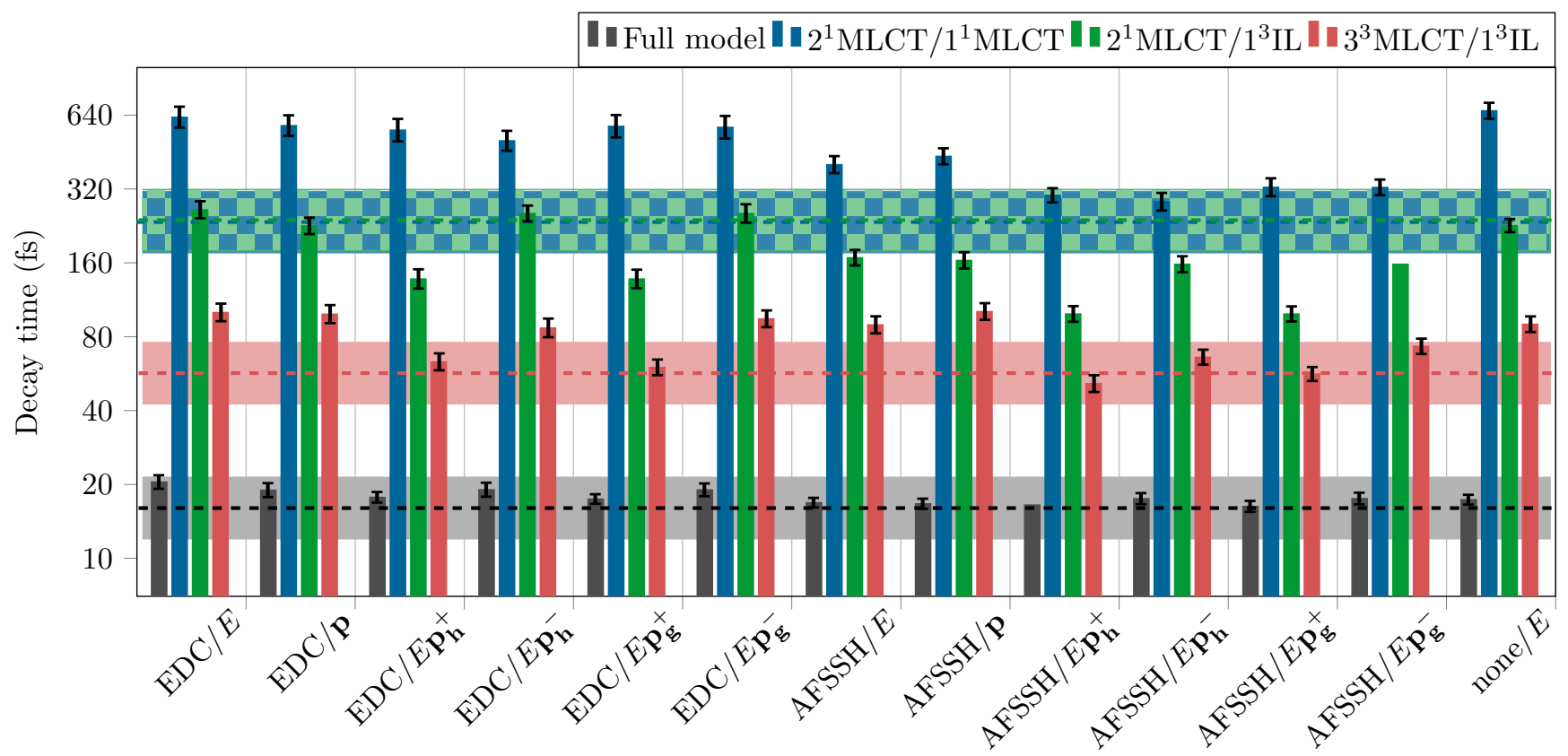

Figure 10: Fitted decay times and error bars for the different models and computational methods plotted on a logarithmic scale. Diabatic populations were computed according to Eq. (13). The dashed lines and highlighted areas indicate MCTDH reference values (note that the reference values for $2^{1} \mathrm{MLCT} / 1^{1} \mathrm{MLCT}$ and $2^{1} \mathrm{MLCT} / 1^{3} \mathrm{IL}$ almost coincide and are, therefore, not distinguishable in this plot).

surface hops. For example, one could use the probability to stay in the active state (i.e., one minus the sum of all the hopping probabilities) as a probability for a stochastic reset rather than resetting after every hop. Alternatively, one could refrain from resetting all states for every surface hop.

Note that the concept of size-consistency / intensivity plays a crucial role for, both, momentum rescaling and the decoherence corrections. We posit that this problem is hidden when computations are performed on smaller model systems and only becomes visible for larger more realistic systems as in this work.

On the optimistic side, we find that for any of the models considered, there is at least one surface hopping protocol that produces good results, revealing that errors are not due to non-local quantum effects - which would invalidate the trajectory approximation as such- but rather to small methodological details of the surface hopping method. This work then illustrates that it is important to obtain a deeper understanding of the effect of these details.

\section{Conclusion}

The purpose of this study was to evaluate the reliability of surface hopping dynamics in the challenging case of transition metal complexes, which are typically characterised by the presence of a high density of electronic states and a large number of crossings among them. For this purpose, we constructed an LVC model of the $\left[\mathrm{Re}(\mathrm{im})(\mathrm{CO})_{3}(\text { phen })\right]^{+}$complex including 15 vibrational normal modes. Simulations were run on the full set of relevant states (2 singlets and 4 triplets) as well as on smaller subsets. Surface hopping simulations were run by varying three parameters in the algorithm -the mode of momentum rescaling, the treatment of frustrated hops, and the decoherence correction- and then compared against an MCTDH reference. It was found that momentum rescaling along the coupling vector outperforms all other methods and that results of almost the same quality were obtained for rescaling along the gradient difference vector. Also a preference for reflecting the momentum after a frustrated hop was found. Nei- 
ther of the two decoherence corrections applied were completely satisfactory but we discussed simple corrections that may be included in the future. In both cases the importance of sizeconsistency/intensivity was highlighted.

The chosen model system might present a worst-case scenario for the surface hopping method due to the high density of states, the interplay of SOC and vibronic coupling, and the interactions of states of different character. But, nonetheless, this paper serves as a reminder that the surface hopping method needs to describe intricate quantum effects and should not be exercised without caution. It also highlights the need for simple, robust, and sizeconsistent schemes for decoherence and momentum rescaling that are generally applicable as well as computationally feasible.

Acknowledgement FP, SM and LG gratefully acknowledge funding from the Austrian Science Fund - Austria (FWF) within project I2883 (DeNeTheor). MF, EG and CD thank the Labex CSC (ANR-10-LABX- 0026_CSC) and the French/Austrian ANR-15-CE29-002701 (DeNeTheor). The electronic structure calculations were performed at the Vienna Scientific Cluster, the High Performance Computer Centre (HPC) of the University of Strasbourg and nodes cluster of the Laboratoire de Chimie Quantique (CNRS/University of Strasbourg).

\section{Supporting Information Avail- able}

Evaluation of timestep lengths (Figure S1). Time-dependent populations of the dynamics simulations for all methods and models (Figure S2-S53). Fitted decay times using Eq. (12), Figure S54. The research data supporting this publication can be accessed via DOI: 10.17028/rd.lboro.c.4493135.v1. ${ }^{80}$

\section{References}

(1) Domcke, W., Yarkony, D. R., Köppel, H., Eds. Conical Intersections. Electronic structure, dynamics and spec- troscopy; Advanced Series in Physical Chemistry; World Scientific, NJ: Singapore, 2004; Vol. 15.

(2) Martínez, T. J. Insights for Light-Driven Molecular Devices from Ab Initio Multiple Spawning Excited-State Dynamics of Organic and Biological Chromophores. Acc. Chem. Res. 2006, 39, 119-126.

(3) Domcke, W., Yarkony, D. R., Köppel, H., Eds. Conical Intersections. Theory, Computation and Experiment; Advanced Series in Physical Chemistry; World Scientific, NJ: Singapore, 2011; Vol. 17.

(4) Plasser, F.; Barbatti, M.; Aquino, A. J. A.; Lischka, H. Electronically excited states and photodynamics: a continuing challenge. Theor. Chem. Acc. 2012, 131, 1073.

(5) Nelson, T.; Fernandez-Alberti, S.; Roitberg, A. E.; Tretiak, S. Nonadiabatic Excited-State Molecular Dynamics: Modeling Photophysics in Organic Conjugated Materials. Acc. Chem. Res. 2014, 47, 1155-1164.

(6) Long, R.; Prezhdo, O. V.; Fang, W. Nonadiabatic charge dynamics in novel solar cell materials. Wiley Interdiscip. Rev. Comput. Mol. Sci. 2017, \%, e1305.

(7) Oberhofer, H.; Reuter, K.; Blumberger, J. Charge Transport in Molecular Materials: An Assessment of Computational Methods. Chem. Rev. 2017, 117, 10319-10357.

(8) Curchod, B. F. E.; Martínez, T. J. Ab Initio Nonadiabatic Quantum Molecular Dynamics. Chem. Rev. 2018, 118, 33053336.

(9) Tully, J. C. Molecular-Dynamics with Electronic-Transitions. J. Chem. Phys. 1990, 93, 1061-1071.

(10) Kubar, T.; Elstner, M. Efficient algorithms for the simulation of non-adiabatic electron transfer in complex molecular systems: application to DNA. Phys. Chem. Chem. Phys. 2013, 15, 5794. 
(11) Mai, S.; Marquetand, P.; González, L. A general method to describe intersystem crossing dynamics in trajectory surface hopping. Int. J. Quantum Chem. 2015, 115, 1215-1231.

(12) Tavernelli, I. Nonadiabatic Molecular Dynamics Simulations: Synergies between Theory and Experiments. Acc. Chem. Res. 2015, 48, 792-800.

(13) Subotnik, J. E.; Jain, A.; Landry, B.; Petit, A.; Ouyang, W.; Bellonzi, N. Understanding the Surface Hopping View of Electronic Transitions and Decoherence. Annu. Rev. Phys. Chem. 2016, 67, 387417.

(14) Wang, L.; Akimov, A.; Prezhdo, O. V. Recent Progress in Surface Hopping: 20112015. J. Phys. Chem. Lett. 2016, 7, 21002112 .

(15) Spencer, J.; Scalfi, L.; Carof, A.; Blumberger, J. Confronting surface hopping molecular dynamics with Marcus theory for a molecular donorâĂŞacceptor system. Faraday Discuss. 2016, 195, 215-236.

(16) Crespo-Otero, R.; Barbatti, M. Recent Advances and Perspectives on Nonadiabatic Mixed Quantum-Classical Dynamics. Chem. Rev. 2018, 118, 7026-7068.

(17) Filatov, M.; Min, S. K.; Kim, K. S. Direct Nonadiabatic Dynamics by Mixed Quantum-Classical Formalism Connected with Ensemble Density Functional Theory Method: Application to trans -Penta2,4-dieniminium Cation. J. Chem. Theory Comput. 2018, 14, 4499-4512.

(18) Landry, B. R.; Falk, M. J.; Subotnik, J. E. Communication: The correct interpretation of surface hopping trajectories: How to calculate electronic properties. $J$. Chem. Phys. 2013, 139.

(19) Zhu, C.; Nangia, S.; Jasper, A. W.; Truhlar, D. G. Coherent switching with decay of mixing: An improved treatment of electronic coherence for non-bornoppenheimer trajectories. J. Chem. Phys. 2004, 121, 7658-7670.

(20) Zhu, C.; Jasper, A. W.; Truhlar, D. G. Non-Born-Oppenheimer Liouville-von Neumann dynamics. Evolution of a subsystem controlled by linear and population-driven decay of mixing with decoherent and coherent switching. $J$. Chem. Theory Comput. 2005, 1, 527-540.

(21) Granucci, G.; Persico, M. Critical appraisal of the fewest switches algorithm for surface hopping. J. Chem. Phys. 2007, 126, 1-11.

(22) Jaeger, H. M.; Fischer, S.; Prezhdo, O. V. Decoherence-induced surface hopping. $J$. Chem. Phys. 2012, 137, 22A545.

(23) Jain, A.; Alguire, E.; Subotnik, J. E. An Efficient, Augmented Surface Hopping Algorithm That Includes Decoherence for Use in Large-Scale Simulations. J. Chem. Theory Comput. 2016, 12, 5256-5268.

(24) Jasper, A. W.; Truhlar, D. G. Improved treatment of momentum at classically forbidden electronic transitions in trajectory surface hopping calculations. Chem. Phys. Lett. 2003, 369, 60-67.

(25) Plasser, F.; Granucci, G.; Pittner, J.; Barbatti, M.; Persico, M.; Lischka, H. Surface hopping dynamics using a locally diabatic formalism: charge transfer in the ethylene dimer cation and excited state dynamics in the 2-pyridone dimer. J. Chem. Phys. 2012, 137, 22A514.

(26) Granucci, G.; Persico, M.; Toniolo, A. Direct semiclassical simulation of photochemical processes with semiempirical wave functions. J. Chem. Phys. 2001, 114, 10608-10615.

(27) Plasser, F.; Crespo-Otero, R.; Pederzoli, M.; Pittner, J.; Lischka, H.; Barbatti, M. Surface Hopping Dynamics 
with Correlated Single-Reference Methods: 9H-Adenine as a Case Study. J. Chem. Theory Comput. 2014, 10, 13951405.

(28) Mai, S.; Atkins, A. J.; Plasser, F.; González, L. The Influence of the Electronic Structure Method on Intersystem Crossing Dynamics. The Case of Thioformaldehyde. J. Chem. Theory Comput. 2019, 15, 3470-3480.

(29) Müller, U.; Stock, G. Surface-hopping modeling of photoinduced relaxation dynamics on coupled potential energy surfaces. J. Chem. Phys. 1997, 10\%, 62306245 .

(30) Landry, B. R.; Subotnik, J. E. How to recover Marcus theory with fewest switches surface hopping: Add just a touch of decoherence. J. Chem. Phys. 2012, 137, $22 \mathrm{~A} 513$.

(31) Chen, H. T.; Reichman, D. R. On the accuracy of surface hopping dynamics in condensed phase non-adiabatic problems. J. Chem. Phys. 2016, $144,094104$.

(32) Käb, G. Fewest switches adiabatic surface hopping as applied to vibrational energy relaxation. J. Phys. Chem. A 2006, 110, $3197-3215$.

(33) Parandekar, P. V.; Tully, J. C. Mixed quantum-classical equilibrium. J. Chem. Phys. 2005, 122.

(34) Topaler, M. S.; Hack, M. D.; Allison, T. C.; Liu, Y.-P.; Mielke, S. L.; Schwenke, D. W.; Truhlar, D. G. Validation of trajectory surface hopping methods against accurate quantum mechanical dynamics and semiclassical analysis of electronic-to-vibrational energy transfer. J. Chem. Phys. 1997, 106, 8699-8709.

(35) Topaler, M. S.; Allison, T. C.; Schwenke, D. W.; Truhlar, D. G. What is the best semiclassical method for photochemical dynamics of systems with conical intersections? J. Chem. Phys. 1998, 109, 3321-3345.

(36) Jasper, A. W.; Hack, M. D.; Truhlar, D. G. The treatment of classically forbidden electronic transitions in semiclassical trajectory surface hopping calculations. J. Chem. Phys. 2001, 115, 1804 1816 .

(37) Mignolet, B.; Curchod, B. F. E. ExcitedState Molecular Dynamics Triggered by Light Pulses-Ab Initio Multiple Spawning vs Trajectory Surface Hopping. J. Phys. Chem. A 2019, 123, acs.jpca.9b00940.

(38) Plasser, F.; Lischka, H. Semiclassical dynamics simulations of charge transport in stacked pi-systems. J. Chem. Phys. 2011, 134, 34309 .

(39) Worth, G. A.; Hunt, P.; Robb, M. A. Nonadiabatic dynamics: A comparison of surface hopping direct dynamics with quantum wavepacket calculations. $J$. Phys. Chem. A 2003, 10\%, 621-631.

(40) Min, S. K.; Agostini, F.; Tavernelli, I.; Gross, E. K. U. Ab Initio Nonadiabatic Dynamics with Coupled Trajectories: A Rigorous Approach to Quantum (De)Coherence. J. Phys. Chem. Lett. 2017, 8, 3048-3055.

(41) Köppel, H.; Domcke, W.; Cederbaum, L. S. Multimode MolecularDynamics Beyond the Born-Oppenheimer Approximation. Adv. Chem. Phys. 1984, 57, 59-246.

(42) Raab, A.; Worth, G. A.; Meyer, H.D.; Cederbaum, L. S. Molecular dynamics of pyrazine after excitation to the $\mathrm{S} 2$ electronic state using a realistic 24-mode model Hamiltonian. J. Chem. Phys. 1999, 110, 936-946.

(43) Krawczyk, R. P.; Malsch, K.; Hohlneicher, G.; Gillen, R. C.; Domcke, W. Conical intersection in trans-butadiene: ultrafast dynamics and optical spectra. Chem. Phys. Lett. 2000, 320, 535-541. 
(44) Faraji, S.; Meyer, H.-D.; Köppel, H. Multistate vibronic interactions in difluorobenzene radical cations. II. Quantum dynamical simulations. J. Chem. Phys. 2008, 129, 074311.

(45) Tamura, H.; Burghardt, I. Ultrafast Charge Separation in Organic Photovoltaics Enhanced by Charge Delocalization and Vibronically Hot Exciton Dissociation. J. Am. Chem. Soc. 2013, 135, 16364-16367.

(46) Eng, J.; Gourlaouen, C.; Gindensperger, E.; Daniel, C. Spin-Vibronic Quantum Dynamics for Ultrafast ExcitedState Processes. Acc. Chem. Res. 2015, 48, 809-817.

(47) Fumanal, M.; Daniel, C. Description of excited states in $[\operatorname{Re}($ Imidazole $)(\mathrm{CO}) 3($ Phen $)]+$ including solvent and spin-orbit coupling effects: Density functional theory versus multiconfigurational wavefunction approach. J. Comput. Chem. 2016, 3, 2454-2466.

(48) Meyer, H.-D.; Manthe, U.; Cederbaum, L. The multi-configurational time-dependent Hartree approach. Chem. Phys. Lett. 1990, 165, 73-78.

(49) Beck, M. H.; Jäckle, A.; Worth, G. A.; Meyer, H.-D. The multiconfiguration time-dependent Hartree (MCTDH) method: a highly efficient algorithm for propagating wavepackets. Phys. Rep. 2000, 324, 1-105.

(50) Meyer, H.-D., Gatti, F., Worth, G. A., Eds. Multidimensional Quantum Dynamics: MCTDH Theory and Applications; Wiley-VCH, 2009.

(51) Plasser, F.; Gómez, S.; Menger, M. F. S. J.; Mai, S.; González, L. Highly efficient surface hopping dynamics using a linear vibronic coupling model. Phys. Chem. Chem. Phys. 2019, 21, 57-59.
(52) Worth, G. A.; Cederbaum, L. S. Beyond Born-Oppenheimer: Molecular dynamics through a conical intersection. Annu. Rev. Phys. Chem. 2004, 55, 127-158.

(53) Fumanal, M.; Gindensperger, E.; Daniel, C. Ultrafast Excited-State Decays in $[\operatorname{Re}(\mathrm{CO}) 3(\mathrm{~N}, \mathrm{~N})(\mathrm{L})] \mathrm{n}+$ : Nonadiabatic Quantum Dynamics. J. Chem. Theory Comput. 2017, 13, 1293-1306.

(54) Fumanal, M.; Gindensperger, E.; Daniel, C. Ligand substitution and conformational effects on the ultrafast luminescent decay of $[\operatorname{Re}(\mathrm{CO}) 3$ (phen $)(\mathrm{L})]+(\mathrm{L}=$ imidazole, pyridine $)$ : non-adiabatic quantum dynamics. Phys. Chem. Chem. Phys. 2018, 20, 1134-1141.

(55) Penfold, T. J.; Gindensperger, E.; Daniel, C.; Marian, C. M. Spin-Vibronic Mechanism for Intersystem Crossing. Chem. Rev. 2018, 118, 6975-7025.

(56) Harabuchi, Y.; Eng, J.; Gindensperger, E.; Taketsugu, T.; Maeda, S.; Daniel, C. Exploring the Mechanism of Ultrafast Intersystem Crossing in Rhenium(I) Carbonyl Bipyridine Halide Complexes: Key Vibrational Modes and Spin-Vibronic Quantum Dynamics. J. Chem. Theory Comput. 2016, 12, 2335-2345.

(57) Fumanal, M.; Plasser, F.; Mai, S.; Daniel, C.; Gindensperger, E. Interstate Vibronic Coupling Constants Between Electronic Excited States for Complex Molecules. J. Chem. Phys. 2018, 124119.

(58) Fumanal, M.; Gindensperger, E.; Daniel, C. Ultrafast Intersystem Crossing vs. Internal Conversion in $\alpha$-diimine Transition Metal Complexes: Quantum Evidence. J. Phys. Chem. Lett. 2018, 9, 5189-5195.

(59) Mai, S.; Marquetand, P.; González, L. Nonadiabatic dynamics: The SHARC approach. Wiley Interdiscip. Rev. Comput. Mol. Sci. 2018, 8, e1370. 
(60) Mai, S.; Plasser, F.; Marquetand, P.; González, L. In Attosecond Molecular Dynamics; Vrakking, M. J. J., Lepine, F., Eds.; Theoretical and Computational Chemistry Series; The Royal Society of Chemistry, 2019.

(61) Mai, S.; Richter, M.; Heindl, M.; Menger, M. F. S. J.; Atkins, A.; Ruckenbauer, M.; Plasser, F.; Oppel, M.; Marquetand, P.; González, L. SHARC2.0: Surface Hopping Including Arbitrary Couplings - Program Package for NonAdiabatic Dynamics. sharc-md.org, 2018.

(62) Schuurman, M. S.; Yarkony, D. R. On the vibronic coupling approximation: A generally applicable approach for determining fully quadratic quasidiabatic coupled electronic state Hamiltonians. J. Chem. Phys. 2007, 127, 094104.

(63) Tegmark, M. Importance of quantum decoherence in brain processes. Phys. Rev. E - Stat. Physics, Plasmas, Fluids, Relat. Interdiscip. Top. 2000, 61, 4194-4206.

(64) Zurek, W. H. Decoherence, einselection, and the quantum origins of the classical. Rev. Mod. Phys. 2003, 75, 715-775.

(65) Subotnik, J. E.; Shenvi, N. A new approach to decoherence and momentum rescaling in the surface hopping algorithm. J. Chem. Phys. 2011, 134, 30-45.

(66) Herman, M. F. Nonadiabatic semiclassical scattering. I. Analysis of generalized surface hopping procedures. J. Chem. Phys. 1984, 81, 754-763.

(67) Coker, D. F.; Xiao, L. Methods for molecular dynamics with nonadiabatic transitions. J. Chem. Phys. 1995, 102, 496-510.

(68) Carof, A.; Giannini, S.; Blumberger, J. Detailed balance, internal consistency, and energy conservation in fragment orbital-based surface hopping. J. Chem. Phys. 2017, 147.
(69) Hillery, M.; O'Connell, R. F.; Scully, M. O.; Wigner, E. P. Distribution functions in physics: Fundamentals. Phys. Rep. 1984, 106, 121-167.

(70) Gill, P. M.; O’Neill, D. P.; Besley, N. A. Two-electron distribution functions and intracules. Theor. Chem. Acc. 2003, 109, 241-250.

(71) Stephens, P. J.; Devlin, F. J.; Chabalowski, C. F.; Frisch, M. J. Ab Initio Calculation of Vibrational Absorption and Circular Dichroism Spectra Using Density Functional Force Fields. J. Phys. Chem. 1994, 98, 11623-11627.

(72) Van Lenthe, E.; Baerends, E. J. Optimized Slater-type basis sets for the elements 1118. J. Comp. Chem. 2003, 24, 11421156 .

(73) Van Lenthe, E.; van Leeuwen, R.; Baerends, E. J.; Snijders, J. G. Relativistic regular two-component Hamiltonians. Int. J. Quantum Chem. 1996, 57, 281-293.

(74) Runge, E.; Gross, E. K. Densityfunctional theory for time-dependent systems. Phys. Rev. Lett. 1984, 52, 9971000 .

(75) Petersilka, M.; Gossmann, U. J.; Gross, E. K. U. Excitation Energies from TimeDependent Density-Functional Theory. Phys. Rev. Lett. 1996, 76, 1212-1215.

(76) Peach, M. J.; Tozer, D. J. Overcoming low orbital overlap and triplet instability problems in TDDFT. J. Phys. Chem. A 2012, 116, 9783-9789.

(77) Wang, F.; Ziegler, T.; van Lenthe, E.; van Gisbergen, S.; Baerends, E. J. The calculation of excitation energies based on the relativistic two-component zeroth-order regular approximation and time-dependent density-functional with full use of symmetry. J. Chem. Phys. 2005, 122, 204103.

(78) Wang, F.; Ziegler, T. A simplified relativistic time-dependent density-functional 
theory formalism for the calculations of excitation energies including spin-orbit coupling effect. J. Chem. Phys. 2005, 123, 154102 .

(79) ADF, SCM, Theoretical Chemistry, Vrije Universiteit,Amsterdam, The Netherlands, 2013, online at https://www.scm.com/Downloads/.

(80) Supporting research data available: Parameters (SHARC input format) and time-dependent populations from the dynamics simulations for all the models evaluated. https://doi.org/10.17028/ rd.lboro.c. 4493135.v1.

(81) Worth, G. A.; Beck, M. H.; Jäckle, A.; Vendrell, O.; Meyer, H.-D. Attosecond Molecular Dynamics; University of Heidelberg, Germany, The MCTDH Package, Version 8.2, (2000). H.-D. Meyer, Version 8.3 (2002), Version 8.4 (2007). O. Vendrell and H.-D. Meyer Version 8.5 (2013). See http://mctdh.uni-hd.de/.

(82) Miao, G.; Bellonzi, N.; Subotnik, J. An extension of the fewest switches surface hopping algorithm to complex Hamiltonians and photophysics in magnetic fields: Berry curvature and "magnetic" forces. $J$. Chem. Phys. 2019, 150.

(83) Mai, S.; Marquetand, P.; González, L. Non-adiabatic and intersystem crossing dynamics in SO 2 . II. The role of triplet states in the bound state dynamics studied by surface-hopping simulations. J. Chem. Phys. 2014, 140, 204302.

(84) Dahl, J. P.; Springborg, M. The Morse oscillator in position space, momentum space, and phase space. J. Chem. Phys. 1988, 88, 4535-4547.

(85) Nangia, S.; Jasper, A. W.; Miller, T. F.; Truhlar, D. G. Army Ants Algorithm for Rare Event Sampling of Delocalized Nonadiabatic Transitions by Trajectory Surface Hopping and the Estimation of Sampling Errors by the Bootstrap Method. J. Chem. Phys. 2004, 120, 3586-3597.
(86) Mai, S.; Marquetand, P.; González, L. Intersystem Crossing Pathways in the Noncanonical Nucleobase 2-Thiouracil: A Time-Dependent Picture. J. Phys. Chem. Lett. 2016, 7, 1978-1983.

(87) Lyakh, D. I.; Musial, M.; Lotrich, V. F.; Bartlett, R. J. Multireference nature of chemistry: the coupled-cluster view. Chem. Rev. 2012, 112, 182-243. 


\section{Graphical TOC Entry}

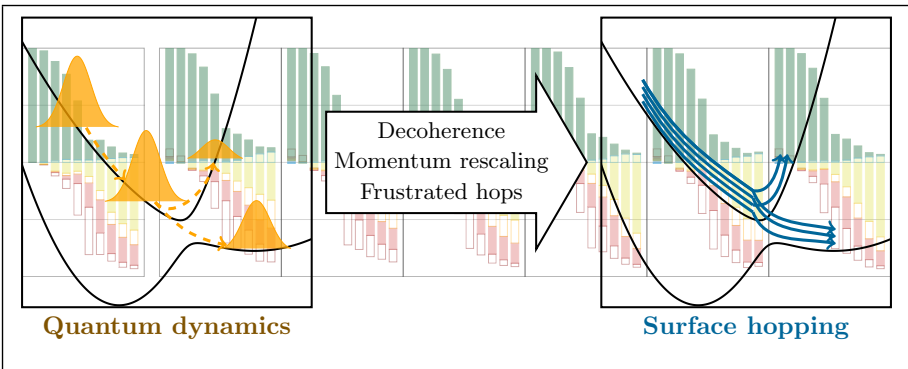

